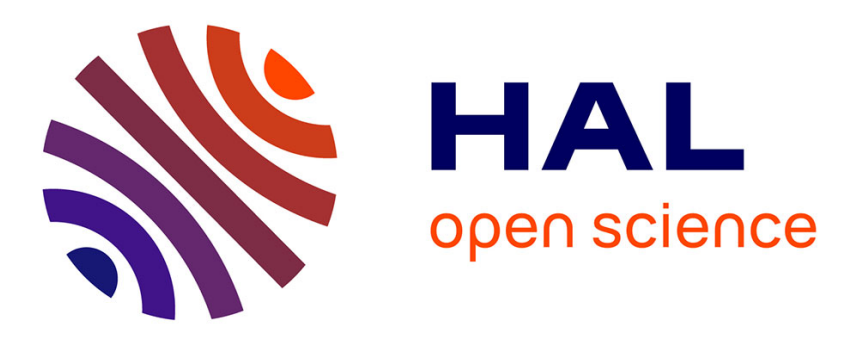

\title{
A comparative multi-property analysis of existing models for the He-N2 potential energy surface
}

Thierry Stoecklin, Anatoli Voronin, Ashok K Dham, Jamie Sanchez-Fortun Stoker, Frederick Mccourt

\section{- To cite this version:}

Thierry Stoecklin, Anatoli Voronin, Ashok K Dham, Jamie Sanchez-Fortun Stoker, Frederick Mccourt. A comparative multi-property analysis of existing models for the He-N2 potential energy surface. Molecular Physics, 2008, 106 (01), pp.75-94. 10.1080/00268970701832363 . hal-00513170

\author{
HAL Id: hal-00513170 \\ https://hal.science/hal-00513170
}

Submitted on 1 Sep 2010

HAL is a multi-disciplinary open access archive for the deposit and dissemination of scientific research documents, whether they are published or not. The documents may come from teaching and research institutions in France or abroad, or from public or private research centers.
L'archive ouverte pluridisciplinaire HAL, est destinée au dépôt et à la diffusion de documents scientifiques de niveau recherche, publiés ou non, émanant des établissements d'enseignement et de recherche français ou étrangers, des laboratoires publics ou privés. 


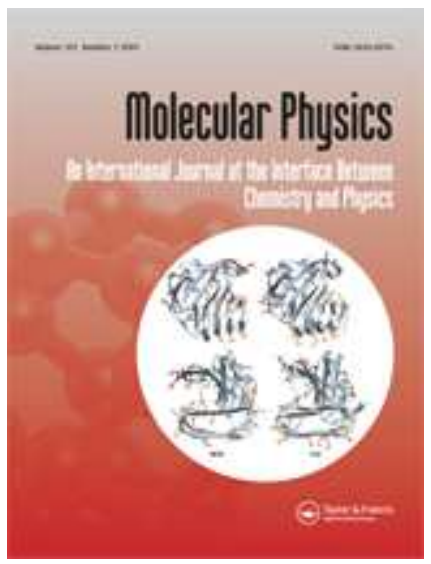

\section{A comparative multi--property analysis of existing models for the He-N2 potential energy surface}

\begin{tabular}{|r|l|}
\hline Journal: & Molecular Physics \\
\hline Manuscript ID: & TMPH-2007-0280.R1 \\
\hline Manuscript Type: & Full Paper \\
\hline Author: & 21-Nov-2007 \\
\hline Complete List of Authors: & $\begin{array}{l}\text { Stoecklin, Thierry; LPCM, Université Bordeaux 1 } \\
\text { voronin, Anatoli; Russian Academy of Sciences, Institute of } \\
\text { Chemical Physics } \\
\text { DHAM, Ashok; Waterloo University, Chemistry } \\
\text { Sanchez-Fortun Stoker, Jamie; Waterloo University, Chemistry } \\
\text { McCourt, Frederick; Waterloo University, Chemistry }\end{array}$ \\
\hline Keywords: & $\begin{array}{l}\text { potential energy surface, inelastic collisions, multi properties } \\
\text { analysis }\end{array}$ \\
\hline & \\
\hline $\begin{array}{l}\text { Note: The following files were submitted by the author for peer review, but cannot be converted } \\
\text { to PDF. You must view these files (e.g. movies) online. }\end{array}$ \\
\hline \begin{tabular}{l} 
TMPH-2007-0280_revised.tex \\
\hline
\end{tabular}
\end{tabular}

\section{s ScholarONE" \\ Manuscript Central}


A comparative multi-property analysis of existing models for the $\mathrm{He}-\mathrm{N}_{2}$ potential energy surface.

2

3

4

5

6

7

8

9

10

11

T. Stoecklin, A. Voronin

Institut des Sciences Moléculaires, 351 cours de la Libération, 33405 Talence Cedex, France.

Ashok K. Dham, ${ }^{1}$ Jamie Sanchez-Fortún Stoker, ${ }^{2}$ and Frederick R. W. McCourt Department of Chemistry, University of Waterloo, Waterloo, Ontario, Canada N2L 3G1

November 21, 2007

\begin{abstract}
The ability of an improved version of a recent three-dimensional ab initio potential energy surface for the He- $\mathrm{N}_{2}$ interaction [Phys. Rev. A 66, 042703 (2002)], determined from high-level coupled-cluster calculations (including full singles and doubles, perturbative triples, and Brueckner orbitals), to predict scattering cross sections and various bulk gas mixture properties is tested. The full three dimensional potential energy surface has been employed for the calculation of vibrational relaxation rates, and a two-dimensional version (at a fixed $\mathrm{N}_{2}$ bond length of $2.0743 a_{0}$ ) has been used for the calculation of molecular beam scattering cross sections using quantum close-coupling
\end{abstract}

\footnotetext{
${ }^{1}$ Permanent address: Department of Physics, Punjabi University, Patiala, 147002, India.

${ }^{2}$ Current address: Chemistry Program, University of Northern British Columbia, 3333 University Way, Prince George, B.C., V2N 4 Z9 Canada.
} 
methods and for the calculation of bulk gas phenomena using classical trajectory methods. The results obtained from the two-dimensional version of the present potential energy surface are compared with those obtained from three other recent accurate two-dimensional representations of the $\mathrm{He}-\mathrm{N}_{2}$ interaction. 


\section{Introduction}

The $\mathrm{He}-\mathrm{N}_{2}$ interaction has served as a prototypical intermolecular interaction, since there is an extensive set of physical phenomena for which measurements exist, and because it is possible to carry out high quality ab initio calculations of the $\mathrm{He}-\mathrm{N}_{2}$ potential energy surface (PES). Indeed, three reasonably accurate $\mathrm{He}-\mathrm{N}_{2}$ potential energy surfaces had already been obtained two decades ago, two from empirical fits to molecular beam scattering and interaction second virial coefficient data $[1,2]$, and the third based upon the semi-empirical Tang-Toennies model [3], with ab initio self-consistent field (SCF) repulsion energies coupled with a damped dispersion series as input to the model [4]. Both empirical potential energy surfaces, namely, the exponential-spline-Morse-spline-Van der Waals (ESMSV) PES of Ref. [1], and the Morse-Morse-Morse-van der Waals (M3SV) PES of Ref. [2], as well as the semi-empirical Bowers-Tang-Toennies (BTT) PES of Ref. [4] have been shown [5] to agree quite well with a large number of bulk transport and relaxation data, and with most of the molecular beam scattering data. It is clear from Ref. [5], however, that all three of these potential energy surfaces have anisotropies that are too weak, especially in the repulsive wall regions.

Partly in response to the conclusion of Ref. [5] regarding the need for a PES with a stronger anisotropy in the repulsive wall region, $\mathrm{Hu}$ and Thakkar [6] employed counterpoisecorrected fourth-order Moeller-Plesset (MP4) calculations of the interaction energy, with the $\mathrm{N}_{2}$ molecule treated as a rigid rotor to obtain the first accurate ab initio PES for the $\mathrm{N}_{2}-$ He interaction. Shortly thereafter, Patel et al. [7] employed counterpoise-corrected basisset-extrapolated coupled-cluster supermolecule calculations at the singles and doubles plus 
perturbative triples, or $\mathrm{CCSD}(\mathrm{T})$, level to obtain a still more accurate rigid-rotor PES. In both of these determinations of the $\mathrm{He}-\mathrm{N}_{2}$ PES, the $\mathrm{N}-\mathrm{N}$ separation was held fixed at the experimental equilibrium bond length of 2.0743 bohr. The MP4 PES of Hu and Thakkar has 61 ab initio points, while the CCSD(T) PES of Patel et al. has 112 ab initio points.

The Hu-Thakkar calculations were extended by Reid et al. [8] to include two additional $\mathrm{N}-\mathrm{N}$ separations in order to be able to calculate vibrational relaxation times associated with the collisional relaxation of $\mathrm{N}_{2}(v=1)$ to $\mathrm{N}_{2}(v=0)$ for comparison with their experimental results $[8,9]$. Their revised $\mathrm{N}_{2}-\mathrm{He}$ PES also included additional points in the neighborhood of the global minimum and is represented by a total of 210 ab initio points. Reid et al. [8] obtained good agreement between results calculated from their stretching-dependent PES using the coupled-states (CS) flag of the Hutson-Green [10] MOLSCAT code suite and the experimental results. They also examined resonances in the energy range over which their calculations were carried out in order to determine their contribution to the vibrational deactivation rate coefficient for $\mathrm{N}_{2}(v=1)$.

As the temperature dependence of the vibrational deactivation rate coefficient for ${ }^{16} \mathrm{O}_{2}(v=$ 1) by ${ }^{3} \mathrm{He}$ at very low temperatures was found by Balakrishnan and Dalgarno [11] to be very unusual at low and very low temperatures, and as the results obtained for the vibrational quenching of $\mathrm{N}_{2}(v=1)$ by ${ }^{3} \mathrm{He}$ obtained by Reid et al. [8] from CS calculations using their MP4-based PES were limited to a higher temperature interval, Stoecklin et al. [12] re-examined the quenching of vibrationally-excited $\mathrm{N}_{2}(v=1)$ by ${ }^{3} \mathrm{He}$ at very low temperatures to see if the behavior observed by Balakrishnan and Dalgarno was specific to $\mathrm{O}_{2}$ or more general. To give as unequivocal an answer as possible, they improved the quality of the stretching-dependent $\mathrm{N}_{2}-\mathrm{He}$ PES both by carrying out extensive CCSD(T)-level quantum 
supermolecule calculations (also incorporating Brueckner orbitals [13]) and by extending the number of ab initio points from 210 to 390 . The ab initio points were fitted to a threedimensional functional representation via the reproducing-kernel Hilbert-space method of Ho and Rabitz [14], which utilizes beta and Gauss hypergeometric functions: the resultant PES is referred to as a BCCD PES. At the same time, in order to obtain a better understanding of the roles of shape and Feshbach resonances on the relaxation process at extremely low temperatures, they carried out close-coupled (CC) quantum scattering calculations using the Newmat quantum scattering code [15] built around the Magnus propagator [16]. Their results for the vibrational quenching cross section of $\mathrm{N}_{2}(v=1, j=0)$ by ${ }^{3} \mathrm{He}$ as a function of total energy showed the same behavior as that found by Balakrishnan and Dalgarno [11] for the vibrational quenching cross section of ${ }^{16} \mathrm{O}_{2}(v=1)$ by ${ }^{3} \mathrm{He}$. Their results also agreed well with the earlier calculations of Reid et al. [8] for total energies of $200 \mathrm{~cm}^{-1}$ and above, but differed by up to two orders of magnitude from the Reid et al. results for total energies below $200 \mathrm{~cm}^{-1}$.

Improvements made to the $\mathrm{BCCD}(\mathrm{T}) \mathrm{PES}$ for the present study are described in Section II of this paper.

At the microscopic level, the BCCD $\mathrm{N}_{2}-\mathrm{He}$ PES, with the $\mathrm{N}-\mathrm{N}$ separation fixed at $2.0743 a_{0}$, has been employed to calculate $j=0 \rightarrow j^{\prime}=2,4$ and $j=1 \rightarrow j^{\prime}=3$ state-tostate differential scattering cross sections at collision energy $27.3 \mathrm{meV}\left(220.2 \mathrm{~cm}^{-1}\right)$, and total differential scattering cross sections at collision energies $27.3 \mathrm{meV}, 31.0 \mathrm{meV}\left(250.0 \mathrm{~cm}^{-1}\right)$, and $70.4 \mathrm{meV}\left(567.8 \mathrm{~cm}^{-1}\right)$. We have also calculated the total integral scattering cross section as a function of speed over the range $0.8-2.6 \mathrm{~km} \mathrm{~s}^{-1}$. These scattering calculations have all been carried out at the quantum coupled-channel (CC) level, using a fully parallelized version 
of the MOLSCAT program suite [17] coupled with the differential scattering code package of Green [18]. Collision energies for the differential scattering cross sections and the speed range for the total integral scattering cross sections have been chosen to coincide with the available experimental state-to-state differential [19], total differential [19,20,21, 1], and total integral $[22,23]$ scattering data.

Equivalent calculations have also been carried out for a number of other $\mathrm{N}_{2}-\mathrm{He}$ potential energy surfaces for comparison with the BCCD results. The specific potential energy surfaces considered for this purpose are: the empirical potential energy surfaces obtained by Beneventi et al. [1], and by Gianturco al. [2] from fittings to experimental data, a recent semi-empirical exchange-Coulomb (XC) model PES [24], the CCSD(T)-level ab initio PES of Patel et al. [7], and the MP4-level ab initio PES of $\mathrm{Hu}$ and Thakkar [6]. The two empirical potential energy surfaces have been included because they were obtained by direct fittings to experimental data, including molecular beam scattering data, while the semi-empirical XC(fit) PES [24] has been included as it appears to give the best overall agreement with the full set of available experimental data.

Because there appear to be significant differences at large scattering angles $\left(120^{\circ} \leq \theta \leq\right.$ $\left.180^{\circ}\right)$ between the state-to-state differential cross sections calculated at collision energy 27.3 meV using the two empirical potential surfaces $[1,2]$ on the one hand, and the semi-empirical $\mathrm{XC}$ (fit) PES [24], previous ab initio potential energy surfaces [6, 7], and the present BCCD PES on the other hand, additional state-to-state differential scattering calculations have been carried out using a code [25] based upon the unrestricted nearside-farside method proposed by McCabe and Connor [26] in order to try to identify those features of the PES that are responsible for the observed differences. These quantum scattering calculations and 
the differential and integral cross section results obtained from them are discussed in Section III below.

Interaction second virial coefficient calculations have been carried out over the temperature range $50 \mathrm{~K} \leq T \leq 1000 \mathrm{~K}$ using a semi-classical code, including first translational and rotational quantum corrections, and classical trajectory (CT) calculations of forty-five effective cross sections that enter into the determination of the transport and relaxation properties of bulk gas mixtures have been obtained. The bulk gas mixture properties for which experimental data are available includes binary diffusion, shear viscosity, thermal conductivity, thermal diffusion, rotational relaxation, vibrational relaxation, and shear viscosity, thermal conductivity and thermal diffusion magnetic field-effects. Comparison is made between the values of these properties calculated using a version of the present BCCD PES, in which the $\mathrm{N}_{2}$ bond length is held fixed at its experimental equilibrium value [27] of 2.0743 $a_{0}$, the experimentally-determined properties, and corresponding values of these properties calculated using the other potential energy surfaces considered in this study. The CT calculations and the temperature dependence of the bulk transport and relaxation phenomena for $\mathrm{N}_{2}-\mathrm{He}$ mixtures are discussed in Section $\mathrm{V}$.

Finally, Section VI presents an overall discussion of the $\mathrm{N}_{2}-\mathrm{He}$ PES and the conclusions at which we have arrived based upon the present in-depth study.

\section{The $\mathrm{N}_{2}-\mathrm{He}$ BCCD Potential Energy Surface.}

As the nature of the ab initio calculations and the fitting procedure methodology are unchanged from those reported previously, both of which have been described in detail in 

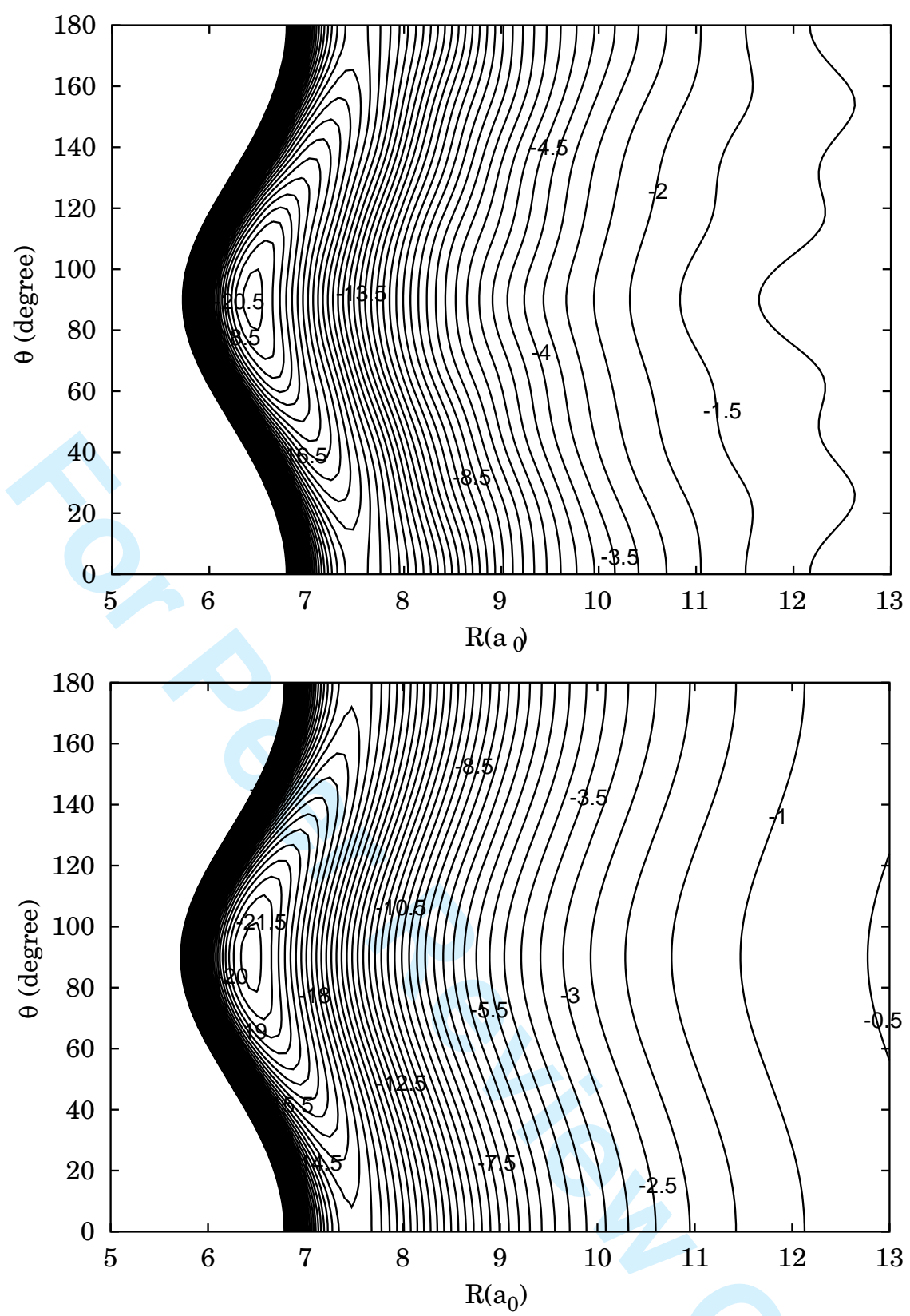

Figure 1: Contour plots illustrating the previous [12] (upper panel) and current (lower panel) rigid-rotor $\left(r=2.0743 a_{0}\right)$ versions of the BCCD PES as functions of interspecies distance $R$ and Jacobi angle $\theta$. The contour lines occur at intervals of $0.5 \mathrm{~cm}^{-1}$.

reference [12], we simply point out those modifications made to the previous version of the BCCD PES. Interaction energies were computed for 390 geometries for the previous version of the BCCD PES [12], with values of the interspecies distance $R$ ranging between $4.0 a_{0}$ 
and $12.0 a_{0}$, the $\mathrm{N}-\mathrm{N}$ separation, $r$, ranging between $1.70 a_{0}$ and $2.65 a_{0}$, and the (Jacobi) angle $\theta$ between the $\mathrm{N}_{2}$ figure axis and the interspecies axis ranging between $0^{\circ}$ and $180^{\circ}$ in steps of $22.5^{\circ}$.
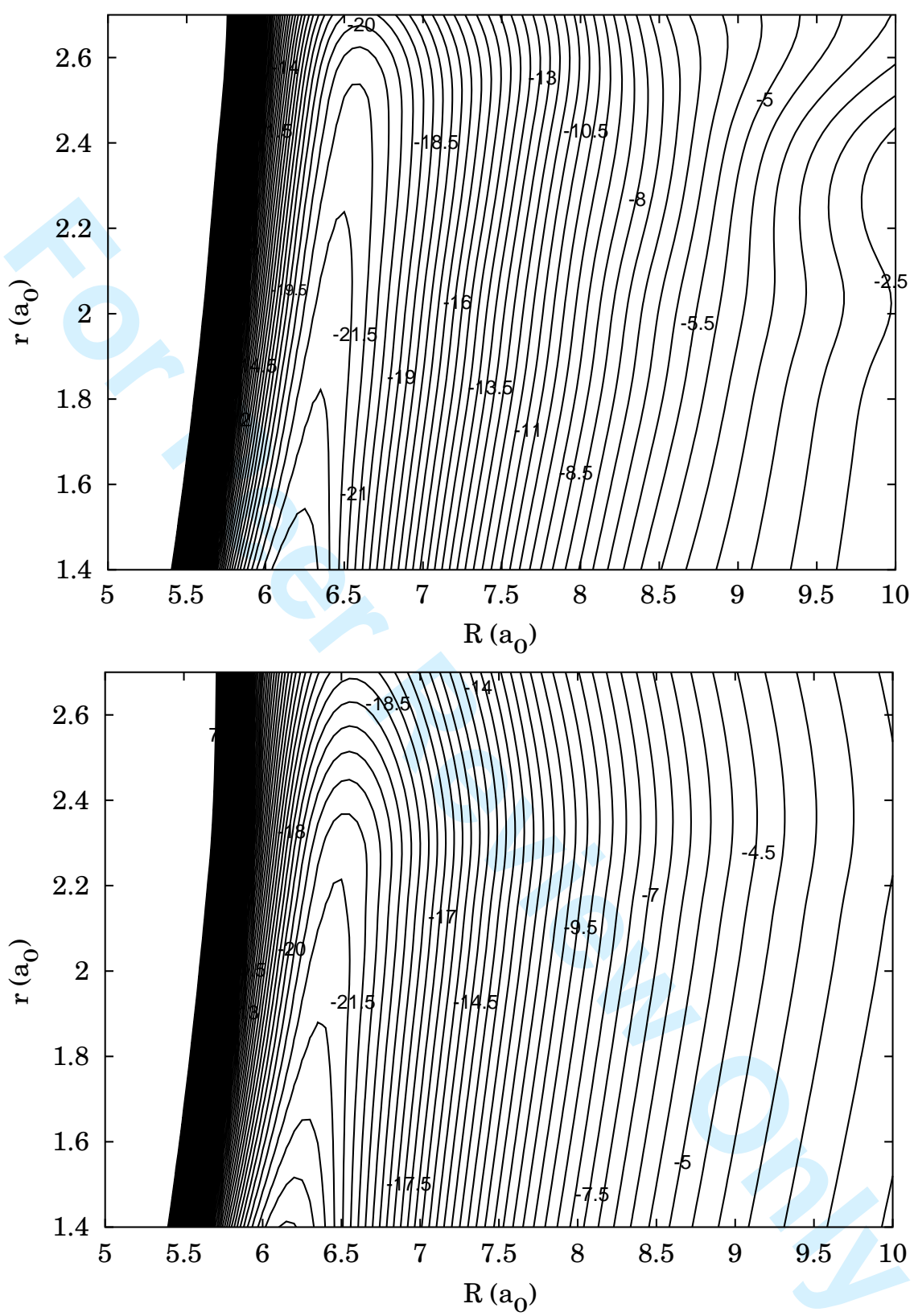

Figure 2: Contour plots illustrating the stretching dependence of the previous [12] (upper panel) and current (lower panel) versions of the full three-dimensional BCCD PES as a function of the interspecies distance $R$ and the N-N separation $r$ for the Jacobi angle $\theta$ fixed at $90^{\circ}$. The contour lines occur at intervals of $0.5 \mathrm{~cm}^{-1}$. 
Two types of improvement have been made to the BCCD PES. The first improvement involves provision of a better description of the $\mathrm{N}_{2}$ vibrational motion through the employment of a finer grid for the $r$ coordinate: this has been achieved by using five values for the $\mathrm{N}-\mathrm{N}$ separation $r$, as previously [12], but in this case restricting them to lie within the interval $1.905 a_{0} \leq r \leq 2.292 a_{0}$. A straightforward calculation of the turning points shows that this grid is more than sufficient to provide the first fourteen vibrational levels of $\mathrm{N}_{2}$ accurately. The second improvement involves obtaining a more accurate representation of both the long- and short-range components of the PES. To accomplish this goal, the range of $R$-values has been extended both inward, from $3 a_{0}$ to $2 a_{0}$, and outward, from $12.0 a_{0}$ to $20.0 a_{0}$, so that the BCCD PES now covers the range $2.0 a_{0} \leq R \leq 20.0 a_{0}$. The set of Jacobi angles is unchanged from that employed in the earlier determination, as the angular dependence is not strongly-varying. As a consequence of these changes to the original grid, the number of points has almost doubled, to 790 points. The diatomic potential [27], which was already very accurate, has not been modified.

The fitting procedure for the new potential energy grid uses the same reproducing kernel (RK) basis set as that employed in Ref. [12], where it is described in detail.

Contour plots illustrating the current rigid-rotor BCCD PES and the previous version [12] of this PES (both for the N-N distance $r$ fixed at its experimental equilibrium value of 2.0743 bohr) are shown in Fig. 1. The global minimum of $-21.73 \mathrm{~cm}^{-1}$ that occurs at $R=$ $6.44 a_{0}, \theta=90^{\circ}$, corresponding to the T-shaped geometry, can clearly be seen from the figure, as can the saddle-point of $-16.43 \mathrm{~cm}^{-1}$ that occurs at $R=7.50 a_{0}, 0^{\circ}$ (equivalently, $180^{\circ}$ ). We note that these values are very close to the values [12] $-21.65 \mathrm{~cm}^{-1}$ at $R=6.4775 a_{0}$ for the global minimum at $\theta=90^{\circ}$ and $-16.20 \mathrm{~cm}^{-1}$ at $R=7.5 a_{0}$ for the saddle-point at 
$\theta=0^{\circ}$ characterizing the previous version of the BCCD PES.

The stretching dependence of the previous and current versions of the BCCD PES is illustrated as a function of $R$ and the $\mathrm{N}-\mathrm{N}$ separation $r$ for the Jacobi angle $\theta$ fixed at $90^{\circ}$ in Fig. 2. The previous version is illustrated in the upper panel and the current version in the lower panel. As can be seen from Fig. 2, the variation of the potential as a function of the stretching coordinate has been improved both in the neighborhood of the equilibrium geometry of the complex and at long range.

\section{Molecular Beam Scattering Cross Sections.}

All differential and total integral cross section calculations reported in this section have been carried out using a parallelized version of the MOLSCAT [17] close-coupled quantum scattering code suite. State-to-state and total differential cross section (DCS) calculations have been performed for the current BCCD PES and for each of the XC(fit) [24], HT [6], MTE [7], ESMSV [1], and M3SV [2] potential energy surfaces at collision energies of 27.3 $\mathrm{meV}, 31.0 \mathrm{meV}$, and $70.4 \mathrm{meV}$. The DCS calculations at $27.3 \mathrm{meV}$ and $31.0 \mathrm{meV}$ collision energy have both been carried out using ${ }^{14} \mathrm{~N}_{2}$ rotational states $j=0$ to $j_{\max }=18$ for the ortho modification of ${ }^{14} \mathrm{~N}_{2}$ and $j=1$ to $j_{\max }=19$ for the para modification (four closed channels for each modification). The maximum total angular momentum quantum number for each of these calculations was $J_{\max }=$ 150. For the calculations at $70.4 \mathrm{meV}$, the corresponding values for $j_{\max }$ were 22 (ortho) and 21 (para), corresponding to three closed channels, while $J_{\max }$ for this collision energy was 200. In addition, total integral cross section calculations have 
been carried out for collision energies ranging from $0.0001 \mathrm{~cm}^{-1}$ to $2345.975 \mathrm{~cm}^{-1}$.

\subsection{Differential Scattering Cross Sections.}

Differential scattering cross section data, obtained from crossed nitrogen and helium beams, have been reported by Faubel et al. [19] at a collision energy of $27.3 \mathrm{meV}$, by Schlemmer [20] at $31.0 \mathrm{meV}$, and by Beneventi et al. [1] at $70.4 \mathrm{meV}$. Faubel et al. [19] measured both the total DCS and the $j=0 \rightarrow j^{\prime}=2, j=1 \rightarrow j^{\prime}=3$, and $j=0 \rightarrow j^{\prime}=4$ rotationally-inelastic state-to-state DCS as functions of the laboratory scattering angle $\Theta$. Their data were then transformed into the center-of-mass frame and reported as functions of the center-of-mass scattering angle $\vartheta$. Both Schlemmer [20] and Beneventi et al. [1] have reported their data only as functions of the laboratory scattering angle $\Theta$.

Comparisons between total DCS data at collision energies of $27.3 \mathrm{meV}$ [19], $31.0 \mathrm{meV}$ [20], and $70.4 \mathrm{meV}[1]$ and total DCS calculated from the present BCCD PES, the XC(fit) [24] PES, and the M3SV [2] PES, are shown in Fig. 3. Because we have insufficient information about the molecular beam experimental conditions to perform a meaningful transformation of our center-of-mass results into the laboratory frame, we have included pseudodata calculated using the M3SV PES for the $31.0 \mathrm{meV}$ comparison and using the ESMSV PES for the $70.4 \mathrm{meV}$ comparison. We have employed the M3SV PES for the $31.0 \mathrm{meV}$ total DCS pseudodata because Faubel [21] found it to provide excellent agreement with the laboratoryframe total DCS data of Schlemmer [20]. Similarly, we have employed the ESMSV PES for the $70.4 \mathrm{meV}$ total DCS pseudodata because the (infinite-order sudden) calculations utilized in the determination of the ESMSV PES from the $70.4 \mathrm{meV}$ total DCS scattering 
data reproduce all experimental points.

The upper panel of Fig. 3 shows the comparison between experimental total DCS data obtained by Faubel et al. [19] for collision energy $27.3 \mathrm{meV}$ (solid symbols) and calculated values obtained from the current BCCD PES (solid curve), the XC(fit) PES (dashed curve), and the M3SV PES (dotted curve). Prior to 1992, it was suspected [2, 5, 28] that the collision energy determination for the scattering data of Faubel et al. [19] might be in error, as no PES was able to reproduce with any degree of accuracy the positions of the diffraction oscillations in their $27.3 \mathrm{meV}$ center-of-mass scattering data, with most calculated cross sections giving diffraction oscillation maxima of the order of a degree forward of the experimental positions. For example, Beneventi et al. [5] found for the ESMSV PES that to achieve a match between the calculated and experimental DCS, the collision energy had to be increased from $27.3 \mathrm{meV}$ to $30.0 \mathrm{meV}$, a change of nearly $10 \%$. It has since been reported (see Ref. [21]) that the angular calibration of the original center-of-mass scattering data of Ref. [19] is incorrect by an average of $5 \%$ of the $\vartheta$-value: we have accounted for this error by plotting the Faubel et al. 27.3 meV DCS values, both total and state-to-state, at angles $\vartheta / 0.95$, with $\vartheta$ denoting the original [19] experimental center-of-mass scattering angle.

The center panel of Fig. 3 compares total DCS values obtained from the present BCCD PES (solid curve), the XC(fit) PES (dashed curve), and pseudodata (open circles) generated from the M3SV PES to represent the experimental data of Schlemmer [20] in the centerof-mass reference frame. The lower panel of Fig. 3 provides a comparison between total DCS values obtained from the present BCCD PES (solid curve), the XC(fit) PES (dashed curve), and pseudodata (open circles) generated from the ESMSV PES for collision energy $70.4 \mathrm{meV}$. 

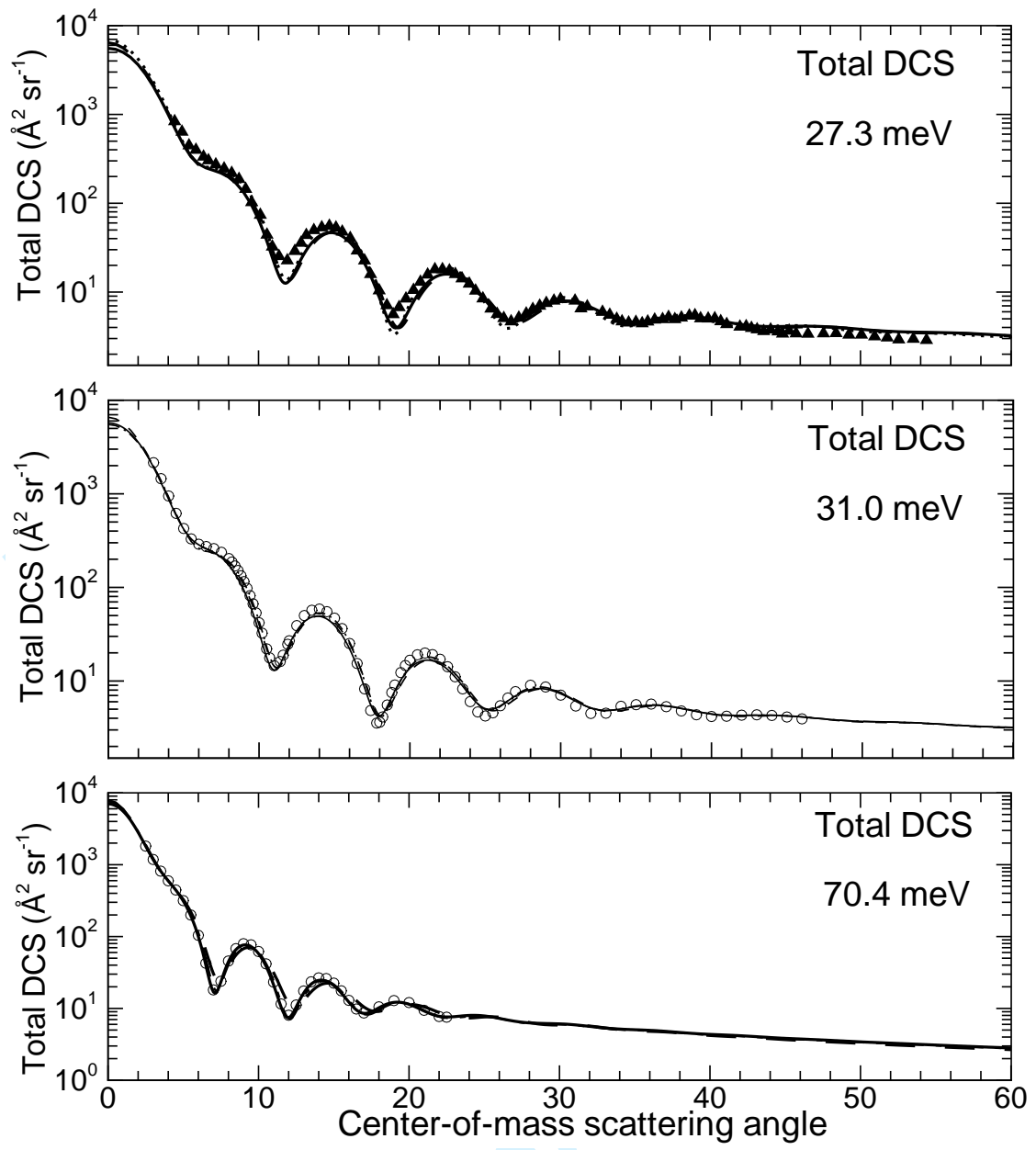

Figure 3: Total differential scattering cross sections for collision energies 27.3, 31.0, and 70.4 meV. Upper panel: experimental data ( $\mathbf{\Delta}$ ), from Ref. [19] (see the text); solid (black) curve, BCCD PES; dashed (red) curve, XC(fit) PES; dotted (blue) curve, M3SV PES. Middle panel: experiment (o), pseudodata generated from the M3SV PES; solid (black) curve, BCCD PES; dashed (red) curve, XC(fit) PES. Lower panel: experiment (o), pseudodata generated from the ESMSV PES; solid (black) curve, BCCD PES; dashed (red) curve, XC(fit) PES; dash-dot (cyan) curve HFD1 PES.

We note in passing that the total DCS calculated using the M3SV PES for collision energy $70.4 \mathrm{meV}$ gives very good agreement with the ESMSV $70.4 \mathrm{meV}$ pseudodata, and if we couple this observation with the fact that the total DCS calculated using the ESMSV PES for collision energy $27.3 \mathrm{meV}$, although not shown in this figure, agrees very well with that calculated from the M3SV PES, we see that these two potential energy surfaces are 
each consistent with the full set of total differential scattering data available. This should, perhaps, not be unexpected, as the M3SV PES was fit in part to the full set of $27.3 \mathrm{meV}$ DCS scattering data, while the ESMSV PES was fit in large part to the $70.4 \mathrm{meV}$ total DCS scattering data. It is gratifying to see that each of these empirical potential energy surfaces predicts equally well the scattering data that were not incorporated into the fitting procedures utilized in the parametrization of the respective potential energy surfaces.
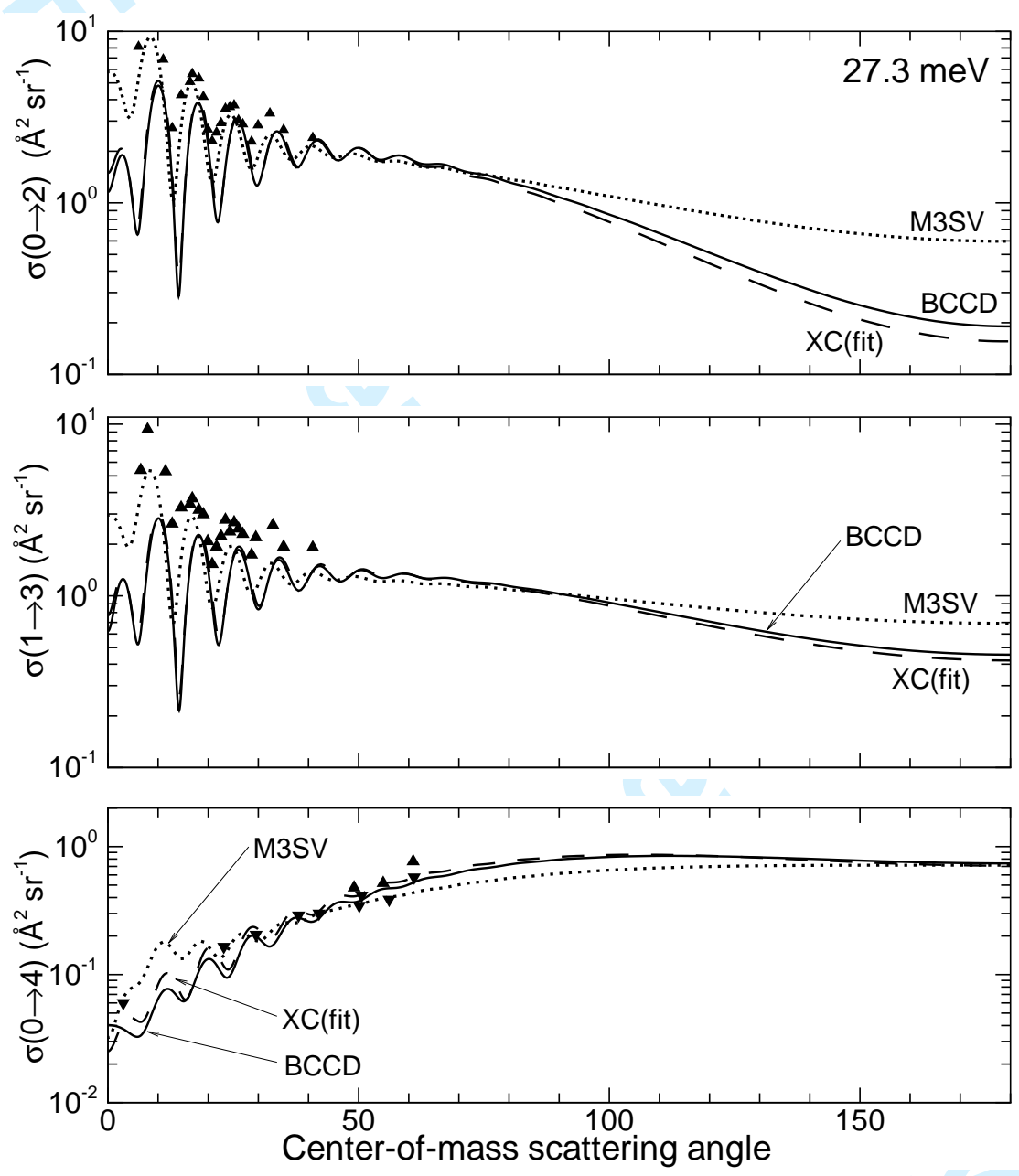

Figure 4: State-to-state differential scattering cross sections for collision energy $27.3 \mathrm{meV}$; the upper panel is for the $0 \rightarrow 2$ inelastic scattering, the middle panel for $1 \rightarrow 3$ inelastic scattering, and the lower panel is for $0 \rightarrow 4$ inelastic scattering. Apart from the data indicated by the symbol $\boldsymbol{\nabla}$, which have been obtained from Fig. 1 of Ref. [28], all experimental data are from Ref. [19]. 
A comparison between calculated and experimental $j=0 \rightarrow j^{\prime}=2, j=1 \rightarrow j^{\prime}=3$, and $j=0 \rightarrow j^{\prime}=4$ state-to-state differential cross sections at a collision energy of 27.3 meV is given in Fig. 4. The top panel shows the $j=0 \rightarrow j^{\prime}=2$ inelastic DCS, the middle panel shows the $j=1 \rightarrow j^{\prime}=3 \mathrm{DCS}$, and the lower panel shows the $j=0 \rightarrow j^{\prime}=4$ DCS. The striking similarity between the $j=1 \rightarrow j^{\prime}=3$ and the $j=0 \rightarrow j^{\prime}=2$ inelastic DCSs for center-of-mass scattering angles $\vartheta$ below $90^{\circ}$ is consistent with $\sigma(1 \rightarrow 3 ; \vartheta)$ being a linear combination of $\sigma(0 \rightarrow 2 ; \vartheta)$ and $\sigma(0 \rightarrow 4 ; \vartheta)$, as has been shown within the infinite-order sudden approximation $[29,30]$. As for the total DCS data, comparison is made between quantum close-coupled calculations of the three state-to-state differential cross sections obtained from the current BCCD PES, the XC(fit) PES, the M3SV PES and the corresponding experimental values [19]. Here, too, there seems to be little to differentiate between the present BCCD PES and the XC(fit) PES, especially for small values $\left(0^{\circ} \leq \vartheta<\right.$ $\left.90^{\circ}\right)$ of the center-of-mass scattering angle. If one were to think in terms of a Legendre expansion of the PES, then it appears that the M3SV has a larger $P_{2}$-anisotropy than does either the BCCD PES or the XC(fit) PES.

We note that for large center-of-mass scattering angles, the M3SV PES (and, similarly, the ESMSV PES) gives results that differ significantly from those obtained with either the $\mathrm{BCCD} \mathrm{PES}$ or the XC(fit) PES, particularly for the $j=0 \rightarrow j^{\prime}=2$ cross section. For this reason, we have carried out a nearside-farside (NF) analysis [26] of the state-to-state DCSs to provide a more detailed examination of the influence of the short- and long-range contributions of the PES to the individual cross sections. The results of this analysis are reported in Sec. IV. 


\subsection{Total Integral Scattering Cross Sections.}

Butz et al. [22] and Candori et al. [23] have both obtained total integral cross section scattering data for the scattering of a beam of velocity-selected ${ }^{4} \mathrm{He}$ atoms by $\mathrm{N}_{2}$ molecules in a scattering chamber maintained at a fixed temperature. In the Butz et al. experiment, the scattering chamber was maintained at $93 \mathrm{~K}$, while in the Candori et al. experiment, the chamber was maintained at $85 \mathrm{~K}$. The earlier data range from ${ }^{4} \mathrm{He}$ speeds of $236 \mathrm{~m} \mathrm{~s}^{-1}$ to $2,600 \mathrm{~m} \mathrm{~s}^{-1}$, and the later data range from $1,500 \mathrm{~m} \mathrm{~s}^{-1}$ to $2,350 \mathrm{~m} \mathrm{~s}^{-1}$. Quantum closecoupled calculations of the total integral cross section have been carried out for total energies ranging from $0.001 \mathrm{~cm}^{-1}$ to nearly $2,350 \mathrm{~cm}^{-1}$ for the BCCD PES and for the ESMSV PES using a parallelized version of the MOLSCAT scattering code [17]. These center-of-mass scattering data need to be energy-averaged, using an appropriate convolution expression, to give laboratory-frame total integral cross sections as a function of ${ }^{4} \mathrm{He}$ speed for a scattering chamber maintained at a specified temperature, $93 \mathrm{~K}$ and $85 \mathrm{~K}$, respectively, for the data of Refs. [22] and [23]. Although small differences are found between the total integral cross section values calculated with the present ab initio BCCD PES, the semi-empirical XC(fit) PES, and the empirical ESMSV PES, they are all significantly smaller than and lie within the experimental uncertainties associated with the experimental measurements reported in Ref. [23]. Corresponding calculations carried out for the ab initio HT and MTE potential energy surfaces give cross section values that are essentially indistinguishable from those obtained with the present BCCD PES. 


\section{Nearside-Farside Analysis for Differential Scattering}

\section{Cross Sections.}

In a series of recent papers, Connor and coworkers $[26,31,32,33]$ adapted the NearsideFarside (NF) method to atom-diatom inelastic scattering and showed that it is an efficient way to extract physical insight from a CC computation of the DCS. This technique was originally introduced into nuclear heavy-ion scattering for Legendre partial-wave series (PWS) by Fuller [34] and later by Hatchell [35]. The NF analysis is based upon the decomposition of the scattering amplitude into two subamplitudes, the Nearside and the Farside amplitudes, that include, respectively, all the scattering from the repulsive and the attractive parts, respectively, of the PES. Structures can arise in the angular distribution from each of the two subamplitudes or from their interferences, and can be analyzed in terms of their links with the different parts of the PES involved. The NF methodology has recently been utilized to give a detailed analysis of the state-to-state inelastic scattering of $\mathrm{N}_{2}$ and $\mathrm{N}_{2}^{+}$by $\mathrm{He}$ [36]. The NF methodology will be utilized herein to analyze the differences between the potential energy surfaces of our comparison set.

We have carried out NF calculations of state-to-state differential scattering cross sections associated with the rotationally inelastic transitions $j=0$ to $j^{\prime}=2,4$ and $j=1$ to $j^{\prime}=3$ for collision energies of $220 \mathrm{~cm}^{-1}$ and $568 \mathrm{~cm}^{-1}$, corresponding to two of the three collision energies, $27.3 \mathrm{meV}$ [19], $31.0 \mathrm{meV}$ [20], and $70.4 \mathrm{meV}$ [1], for which molecular beam differential scattering data are available. Figures 5 through 8 show the nearside $(\mathrm{N})$ and farside $(\mathrm{F})$ components of the three state-to-state cross sections for a selection of the six potential energy surfaces for which calculations have been carried out, namely, the present BCCD PES, the 

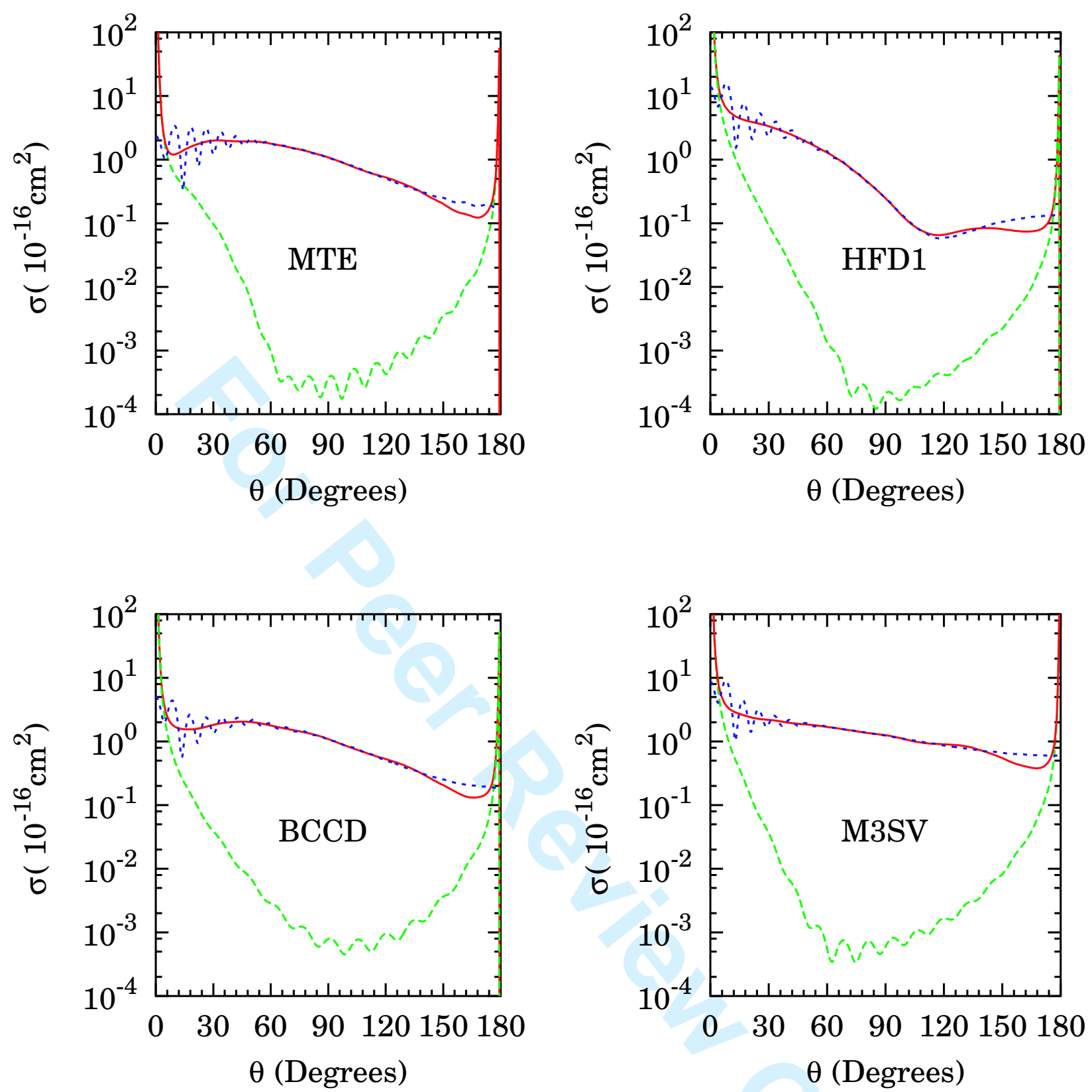

Figure 5: Nearside-Farside analysis of the $j=0 \rightarrow j^{\prime}=2$ state-to-state differential cross sections at total energy $E=220 \mathrm{~cm}^{-1}$; each panel is a semi-log plot of $\sigma(0 \rightarrow 2)$ vs. centerof-mass scattering angle $\vartheta$. Each panel corresponds to a different PES, identified through its acronym; nearside and farside components of the state-to-state DCS are indicated by solid (green) and dash-dot (blue) curves, respectively, and the resultant total state-to-state DCS is indicated by the dashed (red) curve.

ab initio HT [6] and MTE [7] potential energy surfaces, the empirical ESMSV [1] and M3SV [2] potential energy surfaces, and the early semi-empirical HFD1 PES [37]. Figures 5 and 6 

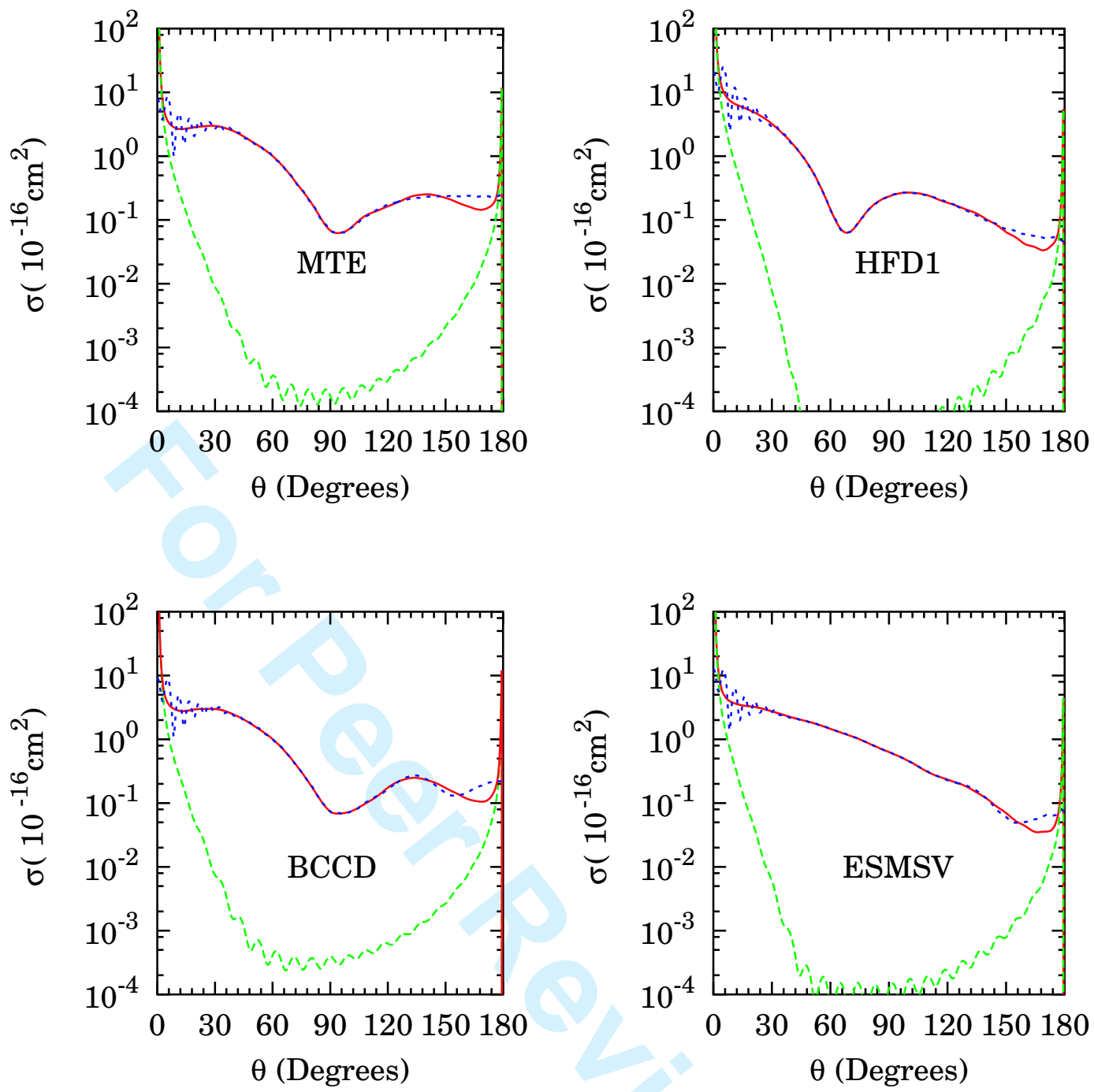

Figure 6: Nearside-Farside analysis of the $j=0 \rightarrow j^{\prime}=2$ state-to-state differential cross sections at total energy $E=568 \mathrm{~cm}^{-1}$; each panel shows a semi-log plot of $\sigma(0 \rightarrow 2)$ vs. the center-of-mass scattering angle $\vartheta$. Each panel corresponds to a different PES, identified through its acronym; nearside and farside components of the state-to-state DCS are indicated by solid (green) and dash-dot (blue) curves, respectively, and the resultant total state-to-state DCS is indicated by the dashed (red) curve.

illustrate the behavior of the $0 \rightarrow 2$ state-to-state DCS at collision energies $220 \mathrm{~cm}^{-1}$ and $568 \mathrm{~cm}^{-1}$, respectively, while Fig. 7 illustrates the behavior of the $0 \rightarrow 4$ state-to-state DCS at both energies for the BCCD and M3SV potential energy surfaces. We have not performed calculations at collision energy $250 \mathrm{~cm}^{-1}$ (corresponding to $31.0 \mathrm{meV}$ ), as any differences be- 
tween results at this collision energy and the results obtained for $220 \mathrm{~cm}^{-1}$ should be rather small. The ab initio MTE PES has been included in these comparisons as it is a precursor PES to the present BCCD PES, while the empirical ESMSV and M3SV potential energy surfaces have been included because they represent best-fits to experimental data. Results for the HT PES, which is an even earlier precursor PES to the present BCCD PES, have not been included in these figures, as they are very similar in general to those obtained for the MTE PES. Finally, the semi-empirical HFD1 PES has been included in the hope that it may provide additional insight into the roles of anisotropies in the short- and long-range regions of a PES, as it is known to be overly anisotropic.

As the energies associated with experiment are almost ten times larger than the well depth of the PES, the farside component of the DCS, which is associated with the attractive part of the PES (the well and long-range parts), is very small in comparison with the nearside component for these energies. This can be seen clearly in each of figures $5-8$, in which the panels on the left-hand side illustrate the results obtained using potential energy surfaces based on extensive grids of ab initio points (MTE and BCCD) and the panels on the right-hand side illustrate the results obtained using one of the empirical potential energy surfaces the M3SV PES (at $E=220 \mathrm{~cm}^{-1}$ ) and ESMSV PES (at $E=568 \mathrm{~cm}^{-1}$ ) and the semi-empirical HFD1 PES.

If we examine firstly Fig. 5, in which the $0-2$ partial DCS is represented for the lowest energy $\left(220 \mathrm{~cm}^{-1}\right)$, we see that while the MTE nearside component is very similar to the BCCD nearside component, the MTE farside component is quite different from that of the BCCD PES. This indicates that the region of the well and the long-range attractive part of the MTE PES differs from the corresponding region for the BCCD PES. We note that at 

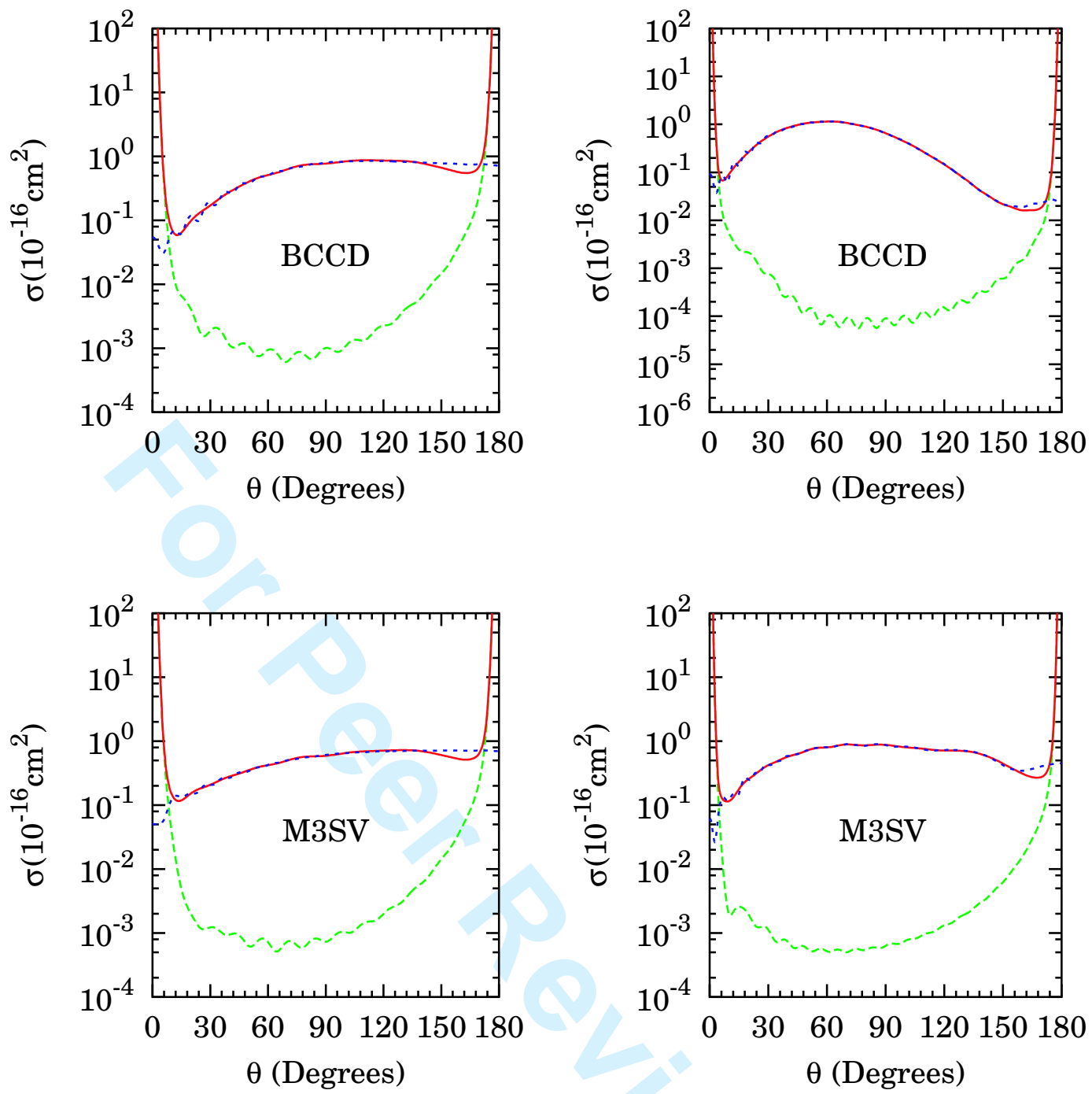

Figure 7: Nearside-Farside analysis of the $j=0 \rightarrow j^{\prime}=4$ state-to-state DCS at total energies $E=220,568 \mathrm{~cm}^{-1}$. Each panel shows a semi-log plot of $\sigma(0 \rightarrow 4)$ vs. the centerof-mass scattering angle $\vartheta$ for a particular PES, identified by its acronym. The panels on the left are for energy $E=220 \mathrm{~cm}^{-1}$ while those on the right are for energy $E=568 \mathrm{~cm}^{-1}$. Each panel corresponds to a different PES, identified through its acronym; nearside and farside components of the state-to-state DCS are indicated by solid (green) and dash-dot (blue) curves, respectively, and the resultant total state-to-state DCS is indicated by the dashed (red) curve.

this energy, the HT PES gives a NS-FS picture that is quite similar to that for the BCCD PES. The relative minimum in the nearside component of the HDF1 PES appearing in the neighborhood of $114^{\circ}$ does not appear to be physical, as none of the other five potential energy 
surfaces gives rise to such a minimum. It is worth noticing that the nearside component obtained from the empirical M3SV PES is larger than that associated with the BCCD PES. The NS and FS results for the ESMSV PES are very similar to those obtained for the M3SV PES, indicating that the short-range components of the empirical model potential energy surfaces are too repulsive. Very similar conclusions can be drawn for the $0 \rightarrow 4$ and $1 \rightarrow 3$ transitions at collision energy $220 \mathrm{~cm}^{-1}$, as can be seen from Fig. 7 for the $0 \rightarrow 4$ transitions, for example. The oscillations in the farside component of the $1 \rightarrow 3$ collision cross section (not shown) are, however, much less pronounced than those otained for the $0 \rightarrow 2$ collision cross section. Note also that interference between the nearside and farside components is clearly visible at small angles in each panel.

If we now turn to Fig. 6 , in which the same comparison is conducted for the $0 \rightarrow 2$ transition at collision energy $568 \mathrm{~cm}^{-1}$, we see that the various potential energy surfaces now behave differently. At this collision energy, only the empirical ESMSV PES does not exhibit a minimum in its nearside component in the neighborhood of $90^{\circ}$. The M3SV PES behaves in a very similar fashion. The repulsive component of the ESMSV PES influencing the collision process at this energy differs from those of the four non-empirical potential energy surfaces in the comparison set. If we now compare the results for the BCCD PES with those for the MTE PES, we see that the farside component of the MTE PES differs from the farside component of the BCCD PES. The M3SV PES and the ESMSV PES give very similar results, as do the HT PES and the MTE PES. Rather similar conclusions can be drawn from an examination of the behavior of the $1 \rightarrow 3$ transition, as it is associated with the same Legendre polynomial expansion coefficients of the intermolecular potential.

In Fig. 7 , we consider the $0 \rightarrow 4$ transition. The MTE PES (not shown) and the BCCD 

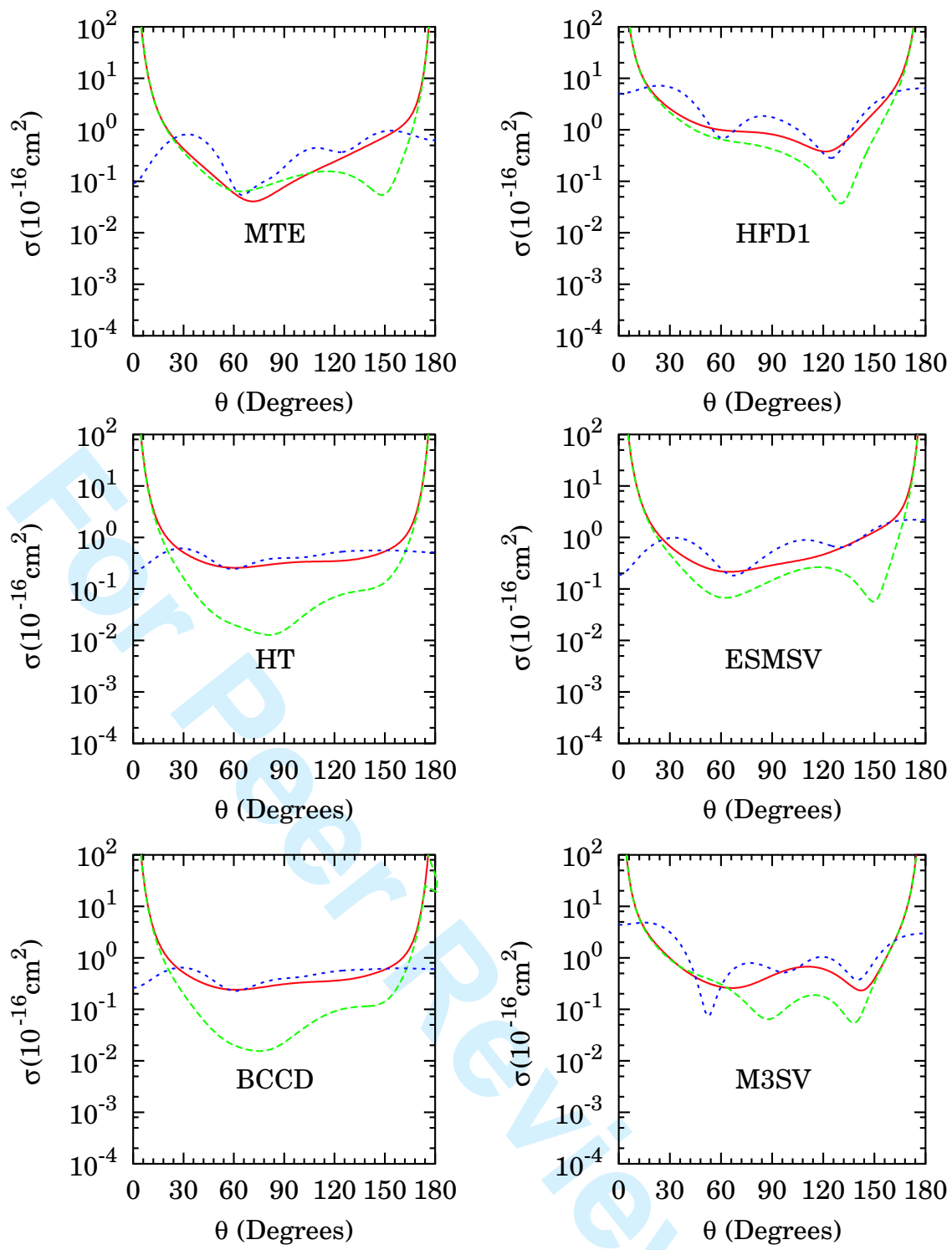

Figure 8: Nearside-Farside analysis of the $j=0 \rightarrow j^{\prime}=2$ state-to-state differential cross sections at total energy $E=13 \mathrm{~cm}^{-1}$; each panel shows a semi-log plot of $\sigma(0 \rightarrow 2)$ vs. the center-of-mass scattering angle $\vartheta$. Each panel corresponds to a different PES, identified through its acronym; nearside and farside components of the state-to-state DCS are indicated by solid (green) and dash-dot (blue) curves, respectively, and the resultant total state-to-state DCS is indicated by the dashed (red) curve.

PES again give very similar results, differing strongly from the results obtained using the ESMSV PES (not shown) and M3SV PES. The curves associated with the HFD1 PES (also not shown) now differ from those obtained with all other models; for example, the 
HFD1 nearside component is the only one that exhibits a minimum for scattering angles in the neighborhood of $90^{\circ}$. This suggests that the short range part of the HFD1 expansion coefficient of the fourth degree Legendre polynomial is quite different from those obtained with all other potential energy surfaces. We see from this discussion that the differences between the various potential energy surfaces can be analysed using the NF analysis of the rotationally selected state-to-state differential cross sections. Unfortunately, however, the energies for which experimental data are available only allow information to be obtained about the repulsive part of the PES.

In order to compare the attractive parts of the various potential energy surfaces more effectively, we have carried out a nearside-farside analysis for two low collision energies, 13 $\mathrm{cm}^{-1}$ and $21 \mathrm{~cm}^{-1}$. The NF analysis of the results of these calculations for energy $13 \mathrm{~cm}^{-1}$ is presented in Fig. 8 for the $j=0 \rightarrow j^{\prime}=2$ DCS. It can be seen from this figure that, as could be anticipated, the attractive farside components now play a much more important role in determining the overall scattering cross section. At $E=13 \mathrm{~cm}^{-1}$, the nearside and farside components are both of the same order of magnitude at all scattering angles, so that the overall result is a general broad oscillatory structure of the scattering cross section as a function of the center-of-mass scattering angle $\vartheta$. Because much the same conclusions can be drawn from the NF analysis for the $j=1 \rightarrow j^{\prime}=3$ DCS for total energy $21 \mathrm{~cm}^{-1}$, those results have not been displayed here. 


\section{Bulk Properties.}

Because bulk gas phenomena are thermally-averaged quantities, they are often considered to be quite insensitive to details of the intermolecular interactions upon which they depend. However, this view has substance only if the temperature range over which measurements of the phenomena are available is limited. Should measurements be available for a particular bulk property over an extensive temperature range, then that bulk property will reflect features of the interaction PES over much of its domain. Nevertheless, were one to wish ultimately to 'invert' bulk data to give a PES, it is best to have data for several different bulk properties over as extensive a temperature range as possible in order to tease out the various features of the PES that are partially masked by the thermal averaging that inevitably enters into the determination of bulk phenomena.

High temperature bulk data are typically sensitive predominantly to the short-range repulsive components of the $\mathrm{PES}$, while low temperature bulk data are generally strongly influenced by the long-range attractive components of the PES. This statement is most readily demonstrated by the temperature dependence of the second virial coefficient: as is well known, the second virial coefficient is negative below the Boyle temperature, $T_{\mathrm{B}}$, while above $T_{\mathrm{B}}$ it is positive, directly reflecting the attractive and repulsive natures of the interaction potential. It is thus perhaps not too surprising that it has been possible to devise a 'direct inversion' procedure to obtain interaction potential functions for monatomic gases from accurate virial data available over a sufficiently extensive temperature range [38]: it may be a little more surprising that it has also been possible to do this for monatomic gases using accurate shear viscosity data [39]. However, it has not yet proven possible to construct 
direct inversion procedures for molecular systems due to the complications introduced by the angular dependence of the PES.

Even though it has not proven possible thus far to construct direct bulk property inversion procedures for molecular systems, accurate bulk property data can be utilized in the role of 'filters' for intermolecular potential energy surfaces. The temperature dependence of the second virial coefficient, the diffusion coefficient, and the shear viscosity coefficient of nonmonatomic gases are all predominantly determined by the spherical component of the PES, and have only relatively small contributions associated with the anisotropic components of the PES. These contributions are typically of the order of $1-5 \%$. For the diffusion and shear viscosity coefficients, this corresponds to inelastic collisional contributions to the effective cross sections [40] that govern their temperature dependence. By contrast, the thermal conductivity coefficient and the thermal diffusion factor are more sensitive to the anisotropic components of the PES, as they depend not only on the translational energy of the molecules, but also on the internal (essentially rotational) energy as well. Inelastic collisional contributions may be of the order of $10-15 \%$ for these phenomena. Finally, bulk relaxation phenomena, such as rotational relaxation, vibrational relaxation, nuclear spin magnetization relaxation, and transport property field-effects [40, 41], depend crucially upon the anisotropic components of the PES in that they do not exist for isotropic systems.

All of the transport and relaxation properties can be expressed in terms of effective cross sections that can be calculated from the PES either via quantum mechanical scattering methods or classical trajectory (CT) methods. A common procedure for utilizing these bulk property measurments in the determination of a PES is to start with a reasonable ab-initiobased PES, carry out forward calculations of the temperature dependence of second virial 
coefficient, and possibly of the diffusion coefficient and/or the shear viscosity coefficient, compare the calculated and experimental values, and then adjust appropriate parameters of the PES. Just such a procedure has been used to determine the $\mathrm{XC}(\mathrm{fit})[24] \mathrm{PES}$ that is a major comparator for the rigid-rotor version of the present ab initio BCCD PES.

Alternatively, if a high-level ab initio PES, such as the present BCCD PES, is available, bulk phenomena can be used to provide effective tests of the predictive power of that ab initio PES. Experimental virial coefficient and ordinary transport property data, with varying degrees of accuracy, are available for comparison purposes. For $\mathrm{N}_{2}-\mathrm{He}$ mixtures specifically, we have, in addition to interaction second virial coefficient data $[42,44,43,45,46,47,48,49]$, diffusion coefficient data $[50,51,52,53,54,55,56,57]$, shear viscosity coefficient data $[58,59,60]$, thermal conductivity coefficient data [61], and thermal diffusion factor data $[62,63,64,65]$. Finally, we have rotational relaxation data $[66,67,68,69,70,71]$, and transport property field-effect data $[72,73,74,75]$.

\subsection{The Bulk Property Calculations.}

Values of the interaction second virial coefficient have been calculated for the rigid-rotor version of the BCCD PES in the semiclassical approximation, including first translational and rotational quantum corrections [76].

The full three-dimensional BCCD PES has been employed for the calculation of the rate of collisional quenching of vibrationally-excited $\mathrm{N}_{2}(v=1, j=0)$ into equilibrium $\mathrm{N}_{2}(v=$ 
0 ), given in terms of the Maxwell average

$$
k_{v j}(T)=\sqrt{\frac{8}{\pi \mu}}\left(k_{\mathrm{B}} T\right)^{-\frac{3}{2}} \int_{0}^{\infty} \sigma_{v j}\left(E_{\mathrm{kin}}\right) \mathrm{e}^{-E_{\mathrm{kin}} / k_{\mathrm{B}} T} E_{\mathrm{kin}} d E_{\mathrm{kin}}
$$

in which $E_{\text {kin }}$ is the relative kinetic energy for a $\mathrm{He}-\mathrm{N}_{2}$ collision, given by

$$
E_{\text {kin }}=\frac{\hbar^{2}}{2 \mu}\left(E-\epsilon_{v j}\right)
$$

with $\mu$ the reduced mass of the $\mathrm{He}-\mathrm{N}_{2}$ collision pair, $E$ the total energy, and $\epsilon_{v j}$ the internal energy of the $\mathrm{N}_{2}$ molecule. The collision cross section $\sigma_{v j}\left(E_{\text {kin }}\right)$, is obtained from the scattering transition operator ( $T$-matrix) elements for transitions of $\mathrm{N}_{2}$ from an initial vib-rotor level labelled by quantum numbers $v, j$ to final levels labelled by quantum numbers $v^{\prime}, j^{\prime}$. A discrete variable representation (DVR) along the Gauss-Hermite grid of the diatomic rovibrational wave functions was calculated by solving the exact diatomic equations using the diatomic potential described in Ref. [12] and a finite-basis representation (FBR) of 150 imaginary exponential wave functions, as described for example by Colbert and Miller [77]. The relevant scattering calculations have been carried out using a code written by one of the authors (Stoecklin): additional calculational details have been given in Ref. [12].

Other bulk property calculations have been carried out for the rigid-rotor version of the BCCD PES, with the $\mathrm{N}-\mathrm{N}$ distance fixed at the $\mathrm{N}_{2}$ experimental equilibrium bond length, $r_{\mathrm{e}}=109.753 \mathrm{pm}$. We do not expect this rigid-rotor approximation to produce any significant differences from values of the bulk properties that would be obtained from the 
full stretching-dependent PES, as all bulk property measurements other than the vibrational relaxation measurements have been carried out either under equilibrium conditions (second virial coefficient measurements) or with only quite small deviations from equilibrium and well within the linear response regime (transport property measurements), and at temperatures $(50 \mathrm{~K}-1000 \mathrm{~K})$ that are well below the characteristic vibrational temperature of $\mathrm{N}_{2}\left(\Theta_{\text {vib }}\right.$ $=3352 \mathrm{~K}$ ), with the consequence that the vast majority of the $\mathrm{N}_{2}$ molecules in the $\mathrm{N}_{2}-\mathrm{He}$ binary mixtures are in their ground vibrational states. This expectation is also supported by quantum close-coupled calculations of the effective cross sections that determine the usual mixture transport coefficients and transport property field-effects for HD-He mixtures $[78,79,80]$ and of similar effective cross sections determining the collision-broadened widths of the $\mathrm{H}_{2}$ Raman lines in $\mathrm{H}_{2}-\mathrm{Ar}$ mixtures [81]. Of equal importance for the determination of the transport coefficients is the fact that a reliable CT code for calculating such effective cross sections for a stretching-dependent PES has yet to be developed.

The effective cross sections that enter the expressions [82] for the transport coefficients of binary mixtures have been obtained from calculations carried out using the CT code package developed by Dickinson and Lee [83, 84] for rigid-rotor-atom collisions. We have employed the same energy mesh utilized in Ref. [24], consisting of fifteen values of the total energy, chosen as pivot points for a Chebychev interpolation on a logarithmic scale, and split into three energy ranges $(0.233 \epsilon, \epsilon),(\epsilon, 33.3 \epsilon)$, and $(33.3 \epsilon-1040 \epsilon)$, with $\epsilon$ representing an appropriate energy unit, chosen here, as in Ref. [24], to be the absolute value of the global minimum of the PES. For the BCCD rigid-rotor PES, the global minimum is $-21.727 \mathrm{~cm}^{-1}$, so that $\epsilon$ has the value $21.727 \mathrm{~cm}^{-1}$. We have employed three points in the lowest-energy interval, nine points in the mid-energy interval, and five points in the highest-energy interval. 
A twelve-point Gaussian quadrature has been employed for the integration over the internal-energy partition parameter [83, 84], and typically 14-20 points have been employed for the integration over the impact parameter, with the integration range split as usual, into two parts. Eight quadrature points have been employed for each of the integrations over the three relevant angular variables. The present choice of quadrature points results in a maximum of 118,000 trajectories at the lowest total energy, and a minimum of 86,000 trajectories at the highest total energy considered, giving rise to a total of more than 1.7 million trajectories for the entire CT calculation for this PES.

\subsection{The Interaction Second Virial Coefficient.}

The interaction second virial coefficient, $B_{\mathrm{AB}}(T)$, for a binary mixture of components gases $A$ and $B$ is related to the mixture and pure component second virial coefficients $B_{\text {mix }}(T), B_{\mathrm{A}}(T), B_{\mathrm{B}}(T)$ by $B_{\text {mix }}(T)=X_{\mathrm{A}}^{2} B_{\mathrm{A}}(T)+2 X_{\mathrm{A}} X_{\mathrm{B}} B_{\mathrm{AB}}(T)+X_{\mathrm{B}}^{2} B_{\mathrm{B}}(T)$, with $X_{\mathrm{A}}, X_{\mathrm{B}}$ the mole fractions of components $\mathrm{A}$ and $\mathrm{B}$.

A comparison between the temperature dependence of the interaction second virial coefficient, $B_{\mathrm{AB}}(T)$, as calculated from the BCCD PES and experiment $[42,44,43,45,46,47,48$, $49]$ is shown in Fig. 9. For comparison purposes, we have included $B_{\mathrm{AB}}(T)$ values calculated from the XC(fit) [24] PES, which has utilized the most accurate of the experimental values [47] in its determination. The HT [6] PES, an earlier ab initio PES obtained from MP4 supermolecule calculations, gives essentially the same results for $B_{\mathrm{AB}}(T)$ over the full range of temperature shown in Fig. 9 for the current BCCD PES. Not surprisingly, the XC(fit) PES, having been fit in part to the accurate data of Brewer and Vaughn [47], provides an 


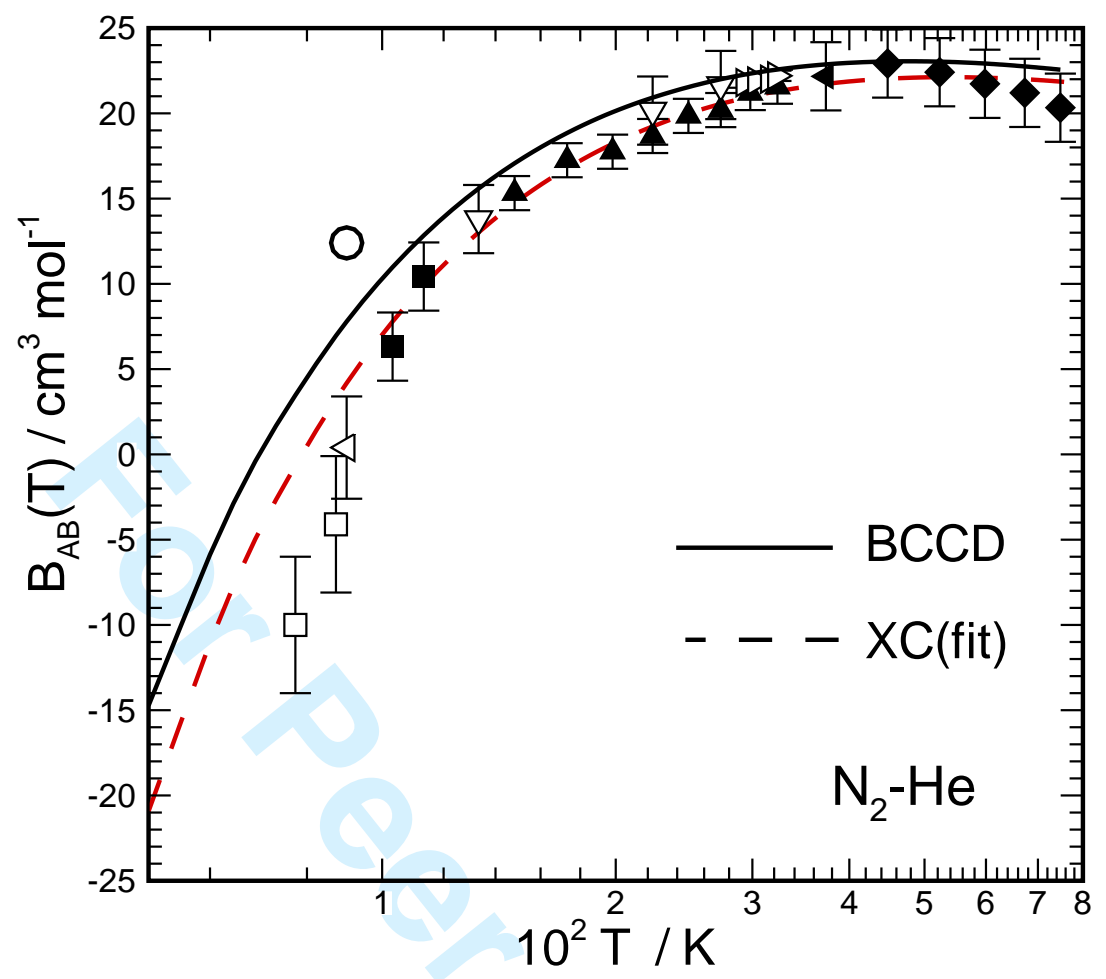

Figure 9: Temperature dependence of the $\mathrm{N}_{2}-\mathrm{He}$ interaction second virial coefficient. Experimental values: $\downarrow$, Ref. [43]; $\mathbf{\Delta}$, Ref. [47]; $\triangleleft$, Ref. [85]; $\square$, Ref. [49]; $\mathbf{\square}$, Ref. [46]; $\nabla$, Ref. [44]; $\bigcirc$, Ref. [42]; ४, Ref. [45]; ๖, Ref. [48].

overall significantly better agreement with the experimental virial data than does either ab initio PES, especially for temperatures above $100 \mathrm{~K}$. Notice, however, that none of these potential energy surfaces shows the same degree of turndown of $B_{\mathrm{AB}}(T)$ for temperatures above $500 \mathrm{~K}$ as can be seen for the experimental values. It remains to be seen whether there is some fundamental feature of the repulsive wall of the PES that all of these potential energy surface determinations lack, or if there is some form of systematic experimental error that has been missed. 


\subsection{Vibrational Relaxation.}

(1)

2

4

5

8
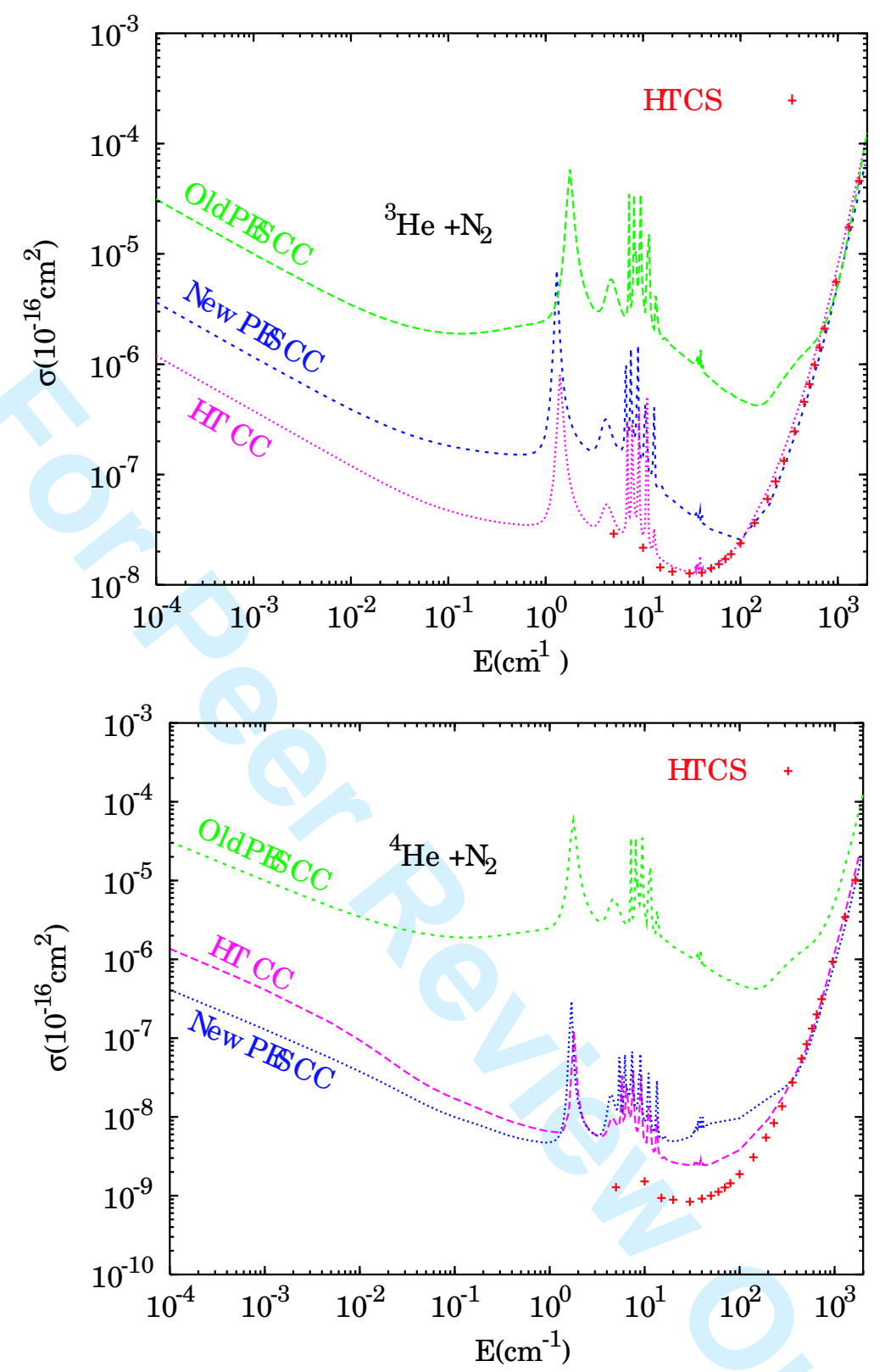

Figure 10: Comparison between the kinetic energy dependence of the vibrational quenching cross section of ${ }^{14} \mathrm{~N}_{2}(v=1, j=0)$ in collision with He obtained using the previous BCCD PES (green curves) [12], the current BCCD PES (blue curves), and the HT PES (mauve

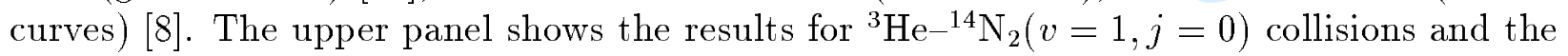
lower panel shows the results for ${ }^{4} \mathrm{He}^{14} \mathrm{~N}_{2}(v=1, j=0)$ collisions. The + symbols (red) represent CS results reported in Ref. [8].

The influence of the new ab initio points that have been added to the repulsive region 
of the BCCD PES on $\mathrm{He}-\mathrm{N}_{2}$ vibrational relaxation is illustrated in Fig. 10, in which the ${ }^{3} \mathrm{He},{ }^{4} \mathrm{He}^{14}{ }^{14} \mathrm{~N}_{2}(v=1, j=0)$ vibrational quenching cross sections obtained from CC calculations employing (the full, i.e. three-dimensional version of) the present BCCD PES are compared with the results obtained from both the previous version [12] of the full BCCD PES and with the Coupled States (CS) approximation results obtained by Reid et al. [8] using the full HT PES. Quantum close-coupled calculations have also been performed at very low energies in order to illustrate the limits of the Wigner regime, in which the cross section varies as the reciprocal of the wavenumber $k$ (given by $E_{k}=\hbar^{2} k^{2}$ ). Figure 10 , taken as a whole, shows clearly that the previous version of the full BCCD PES, with a grid of points starting only at 4 bohr was overly restrictive, and is too repulsive, as the results obtained with the present version of the full BCCD PES are at least an order of magnitude smaller than the results reported in Ref. [12] for collision energies below $300 \mathrm{~cm}^{-1}$.

The upper panel in Fig. 10 illustrates the relative kinetic energy dependence of the vibrational quenching cross section for collisions of ${ }^{3} \mathrm{He}$ with ${ }^{14} \mathrm{~N}_{2}(v=1, j=0)$ obtained for each full PES. The results for all three potential energy surfaces are in good agreement for collision energies above about $800 \mathrm{~cm}^{-1}$; the results for the present version of the full BCCD PES and the full HT PES, both using CC and CS calculations, remain in good agreement down to a collision energy of approximately $100 \mathrm{~cm}^{-1}$. Below collision energy $100 \mathrm{~cm}^{-1}$, however, the results for the present full BCCD PES and the full HT PES differ significantly. How much does the use of the CS approximation for the calculations with the HT PES contribute to this difference? Partly to answer this question, and partly for completeness, we have carried out CC calculations with the full HT PES. Direct comparison of these new $\mathrm{CC}$ results, especially at the lowest collision energies, with the CS approximation calculations 

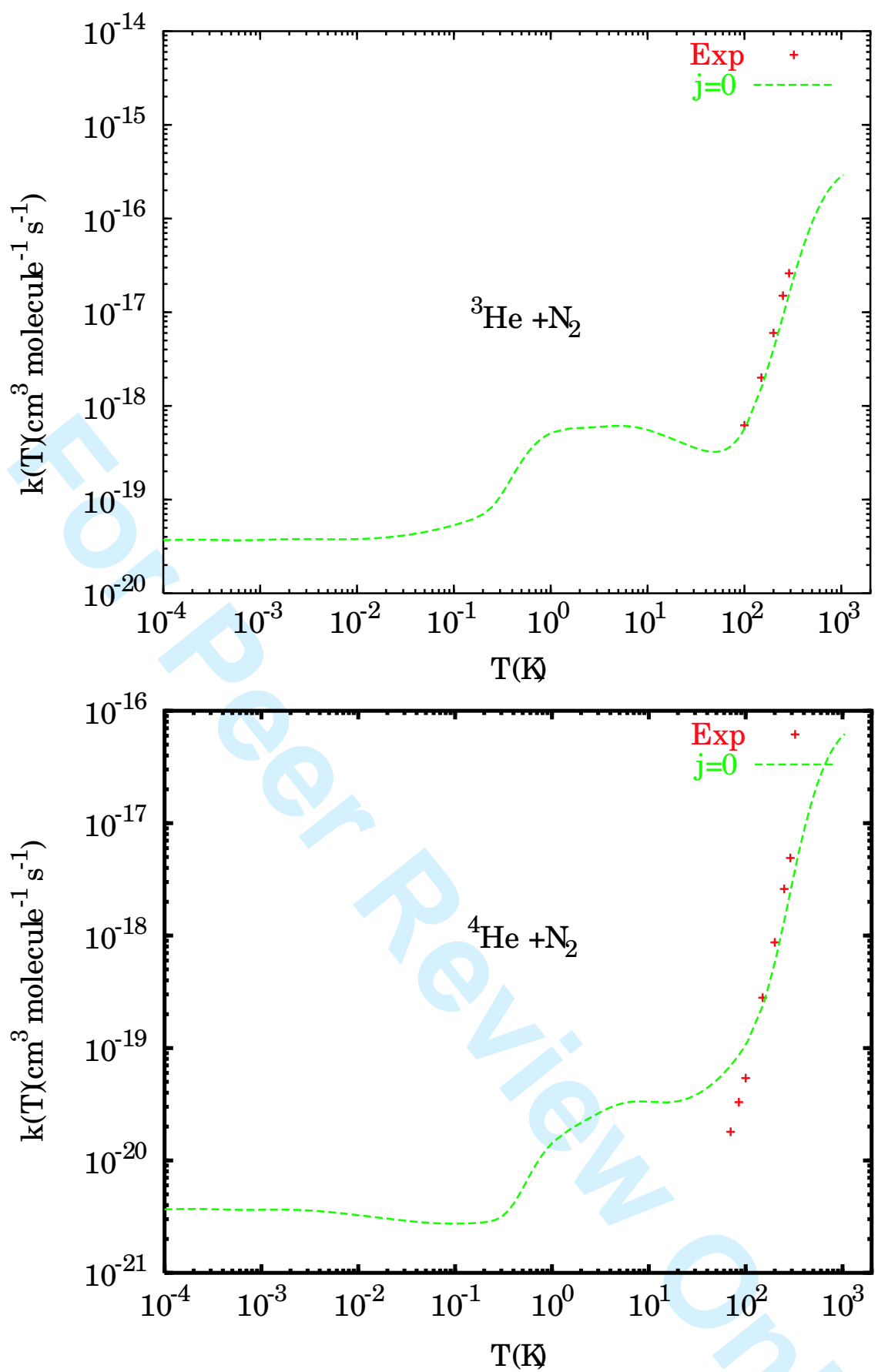

Figure 11: Comparison between the temperature dependence of the vibrational quenching rates for ${ }^{14} \mathrm{~N}_{2}(v=1, j=0)$ in collision with ${ }^{3} \mathrm{He}$ and ${ }^{4} \mathrm{He}$, obtained obtained using the present full BCCD PES and the experimental results of Ref. [8], represented by the + symbols. Upper panel: ${ }^{3} \mathrm{He}$ results, lower panel: ${ }^{4} \mathrm{He}$ results. 
reported in Ref. [8] indicates that CS calculations fare quite well for ${ }^{3} \mathrm{He}^{-14} \mathrm{~N}_{2}(v=1, j=0)$ collisions. The missing resonance structure around collision energy $100 \mathrm{~cm}^{-1}$ may be due more to the energy grid chosen in this region than to the CS approximation itself.

If we turn to the lower panel of Fig. 10, we see that the difference between the vibrational quenching cross section for the previous and present versions of the full BCCD PES for ${ }^{4} \mathrm{He}^{-}$ ${ }^{14} \mathrm{~N}_{2}(v=1, j=0)$ collisions is rather similar to that found for the ${ }^{3} \mathrm{He}$ case, i.e., the previous version of the full BCCD PES is too repulsive, giving quenching cross values that are more than an order of magnitude greater than the values obtained with the present version. It is interesting to note that the quenching cross section values obtained from $\mathrm{CC}$ calculations employing the present version of the full BCCD PES gives much closer agreement with the CC calculations employing the full HT PES of Reid et al. [8] than is obtained (see the upper panel of the figure) for the analogous ${ }^{3} \mathrm{He}$ interaction. Further, we see that the CS results reported in Ref. [8] differ significantly from the present $\mathrm{CC}$ results in this case. As the CS approximation is strictly valid only for 'high' collision speeds, this difference in the performance of the CS approximation for the two isotopomeric collision systems may be the result of the change in the reduced mass of the collision pair from $2.723 \mathrm{amu}$ for the ${ }^{3} \mathrm{He}$ case, versus 3.502 amu for the ${ }^{4} \mathrm{He}$ case: if we recall that the collision energy is very low, it may not be so surprising that there is such a difference for so small a change in the reduced mass of the collision pair.

For temperatures above $100 \mathrm{~K}$, corresponding to temperatures for which experimental values $[8,9]$ of the ${ }^{3} \mathrm{He}^{-14} \mathrm{~N}_{2}(v=1, j=0)$ vibrational quenching cross section are available, the values of the thermally-averaged quenching cross section are dominated by the energy dependence of the quenching cross section in the range $100-1000 \mathrm{~cm}^{-1}$, which is the energy 
range for which the calculations using the present full BCCD PES and the full HT PES are in good agreement: this indicates that the vibrational quenching rate coefficient obtained using the new full BCCD PES will also be in very good agreement with experiment, as can be seen from the upper panel of Fig. 11. For the ${ }^{4} \mathrm{He}^{14} \mathrm{~N}_{2}(v=1, j=0)$ vibrational quenching rate shown in the lower panel of Fig. 11, we see that although good agreement with experiment is obtained for temperatures above $125 \mathrm{~K}$, the calculated values exceed the experimental values significantly for temperatures $100 \mathrm{~K}$ and lower. We note also that the small but distinct minimum in $k(T)$ that occurs at approximately $15 \mathrm{~K}$ for ${ }^{3} \mathrm{He}^{-}-{ }^{14} \mathrm{~N}_{2}$ has become a broad inflection, occurring at approximately the same temperature, for ${ }^{4} \mathrm{He}^{-}{ }^{14} \mathrm{~N}_{2}$. Finally, we see from Fig. 11 that the temperature dependence of $k(T)$ for the $\mathrm{He}-\mathrm{N}_{2}$ system exhibits all three characteristic temperature regimes: the Wigner regime below $10^{-1} \mathrm{~K}$, in which the rate coefficient is constant, an intermediate van der Waals regime, in which the temperature dependence of $k(T)$ is nonmonotonic, and a rovibrational-translational energy transfer regime, in which the $k(T)$ increases monotonically with increasing temperature. We note further that the nonmonotonic behavior of $k(T)$ is associated with the resonances exhibited in the energy dependence of $\sigma(E)$ shown in Fig. 10.

\subsection{Effective Cross Sections and Bulk Transport-Relaxation Phe- nomena.}

The bulk gas transport properties and their magnetic field-effects are governed by sets of temperature-dependent effective cross sections: details concerning notation and the various conventions are as given in Ref. [40,41]. For binary mixtures in which one species is 
monatomic, the other nonmonatomic, the nonmonatomic species is designated by $\mathrm{A}$, and the monatomic species by B. Generalized effective cross sections determined by the unlike-species interaction are designated by $\mathfrak{S}\left(\left.\begin{array}{c}p q s t \\ p^{\prime} q^{\prime} s^{\prime} t^{\prime}\end{array}\right|_{1} ^{\mathbf{k}}\right)_{\mathrm{AB}}$, with $p, q, s, t, p^{\prime}, q^{\prime}, s^{\prime}, t^{\prime}$ being integers denoting the tensorial ranks of velocity $\left(p, p^{\prime}\right)$ and rotational angular momentum $\left(q, q^{\prime}\right)$ polyadics in an expansion of the nonequilibrium distribution function for the gas mixture, while $s, s^{\prime}$ and $t, t^{\prime}$ denote the degrees of orthogonal (associated Laguerre) polynomials in the squares of velocity and angular momentum, respectively, of the accompanying scalar dependences; $\mathrm{k}, 1$ denote the chemical species A, B (here $\mathrm{N}_{2}$, He, respectively).

The binary diffusion coefficient, $D_{\mathrm{AB}}$, is largely determined by the effective cross section $\mathfrak{S}(1000 \mid \mathrm{A})_{\mathrm{AB}}$, while $\mathfrak{S}(2000 \mid \mathrm{A})_{\mathrm{AB}}$ and $\mathfrak{S}\left(\left.{ }_{2000}^{2000}\right|_{\mathrm{B}} ^{\mathrm{A}}\right)_{\mathrm{AB}}$ make the largest contributions to the mixture shear viscosity coefficient, $\eta_{\mathrm{mix}}, \mathfrak{S}(1010 \mid \mathrm{A})_{\mathrm{AB}}, \mathfrak{S}(1001 \mid \mathrm{A})_{\mathrm{AB}}, \mathfrak{S}\left(\left.\begin{array}{l}1001 \\ 1010\end{array}\right|_{\mathrm{A}} ^{\mathrm{A}}\right)_{\mathrm{AB}}$, and $\mathfrak{S}\left(\left.\begin{array}{c}1001 \\ 1010\end{array}\right|_{\mathrm{B}} ^{\mathrm{A}}\right)_{\mathrm{AB}}$, dominate the mixture thermal conductivity coefficient, $\lambda_{\text {mix }}$ and, together with $\mathfrak{S}\left(\left.\begin{array}{c}1010 \\ 1000\end{array}\right|_{\mathrm{A}} ^{\mathrm{A}}\right)_{\mathrm{AB}}$ and $\mathfrak{S}\left(\left.\begin{array}{c}1001 \\ 1000\end{array}\right|_{\mathrm{A}} ^{\mathrm{A}}\right)_{\mathrm{AB}}$, largely determine the thermal diffusion coefficient, $D_{\mathrm{T}}$. Values for effective cross sections of the type $\mathfrak{S}\left(\left.\begin{array}{c}p q s t \\ p^{\prime} q^{\prime} s^{\prime} t^{\prime}\end{array}\right|_{\mathrm{A}} ^{\mathrm{A}}\right)_{\mathrm{AB}}$ can be obtained from tabulations given in Ref. [86], while effective cross sections typified by $\mathfrak{S}\left(\left.{ }_{2000}^{2000}\right|_{\mathrm{B}} ^{\mathrm{A}}\right)_{\mathrm{AB}}, \mathfrak{S}\left(\left.{ }_{1010}^{1001}\right|_{\mathrm{B}} ^{\mathrm{A}}\right)_{\mathrm{AB}}$, and $\mathfrak{S}(1010 \mid \mathrm{B})_{\mathrm{AB}}$, which have not been included in the tabulations of Ref. [86] may be constructed from the effective cross sections listed via exact relations amongst the various effective cross sections, many of which have been given explicitly in Ref. [41].

The most efficacious manner of comparing the calculated effective cross sections determining the field-free transport properties is through a direct comparison between the exper- 
Table 1: Dimensionless root-mean-square deviations for virial and transport property data.

\begin{tabular}{lcccccc}
\hline \multirow{2}{*}{ Bulk Property } & & \multicolumn{5}{c}{ Potential Energy Surface } \\
& Symbol & Data & XC(fit) & BCCD & HT $^{a}$ & MTE $^{a}$ \\
\hline \hline Virials & $B_{\mathrm{AB}}$ & 26 & 0.58 & 1.23 & 1.29 & 1.11 \\
Diffusion & $D_{\mathrm{AB}}$ & 60 & 2.89 & 3.08 & 3.63 & 2.87 \\
Viscosity & $\eta_{\text {mix }}$ & 81 & 1.88 & 2.98 & 4.21 & 2.11 \\
Thermal Conductivity & $\lambda_{\text {mix }}$ & 3 & 1.86 & 2.14 & 2.44 & 1.93 \\
\hline All Experiments & & 170 & 2.18 & 2.73 & 3.67 & 2.30 \\
\hline \hline
\end{tabular}

imentally measured and calculated properties (similarly for second virial coefficients). This can be achieved most transparently by determining the dimensionless root-mean-square deviations (DRMSD) between calculated and measured values. DRMSD values can be defined generically for property $\mathcal{P}$ as

$$
\operatorname{DRMSD}(\mathcal{P}) \equiv\left[\frac{1}{N} \sum_{i=1}^{N}\left(\frac{\mathcal{P}_{i}^{\text {calc }}-\mathcal{P}_{i}^{\text {expt }}}{u_{i}}\right)^{2}\right]^{\frac{1}{2}}
$$

in which $\mathcal{P}_{i}^{\text {calc }}$ and $\mathcal{P}_{i}^{\text {expt }}$ are the calculated and experimentally determined values for property $\mathcal{P}, N$ is the number of data in the comparison set, and $u_{i}$ is the experimental uncertainty assigned to the measured value $\mathcal{P}_{i}^{\text {expt }}$. Table 1 gives the number of data for each property and lists the DRMSD values for each property. By treating the entire set of experimental data as a single set of data, we can obtain an overall DRMSD value for the collection of commonly measured bulk phenomena. The DRMSD values for three comparator potential energy surfaces, XC(fit) [24], HT [6], and MTE [7], also listed, have been taken from Ref. [24]. A DRMSD value of 1 (or less) means that the calculated values all lie within (or well within) the experimental uncertainties, and values greater than 1 are indicators of the level 
Table 2: Comparison between calculated and experimental values for relaxation and production effective cross sections (units $\AA^{2}$ ).

Potential energy surface

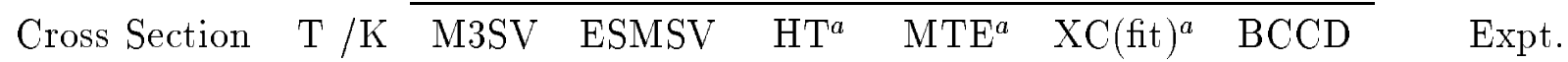

\begin{tabular}{|c|c|c|c|c|c|c|c|c|}
\hline $\mathfrak{S}(0001 \mid \mathrm{A})_{\mathrm{AB}}$ & 304.0 & 2.90 & 2.29 & 4.27 & 4.28 & 4.38 & 4.25 & $4.2 \pm 1.6$ \\
\hline $\mathfrak{S}(0200 \mid \mathrm{A})_{\mathrm{AB}}$ & $\begin{array}{r}77.3 \\
293.0 \\
295.0\end{array}$ & $\begin{array}{l}7.14 \\
3.05 \\
3.04\end{array}$ & $\begin{array}{l}6.30 \\
2.40 \\
2.40\end{array}$ & $\begin{array}{l}7.54 \\
4.34 \\
4.33\end{array}$ & $\begin{array}{l}7.70 \\
4.37 \\
4.36\end{array}$ & $\begin{array}{l}7.77 \\
4.46 \\
4.45\end{array}$ & $\begin{array}{l}7.65 \\
4.34 \\
4.33\end{array}$ & $\begin{array}{l}8.3 \pm 0.9^{c} \\
5.6 \pm 0.3^{c} \\
4.9 \pm 0.5^{d}\end{array}$ \\
\hline $\mathfrak{S}(0 \widehat{2} 00 \mid \mathrm{A})_{\mathrm{AB}}$ & $\begin{array}{l}120.0 \\
186.0 \\
293.0 \\
295.0\end{array}$ & $\begin{array}{r}11.39 \\
9.06 \\
7.36 \\
7.34\end{array}$ & $\begin{array}{r}10.66 \\
8.24 \\
6.50 \\
6.48\end{array}$ & $\begin{array}{r}10.97 \\
9.45 \\
8.31 \\
8.29\end{array}$ & $\begin{array}{r}11.22 \\
9.57 \\
8.34 \\
8.33\end{array}$ & $\begin{array}{r}11.25 \\
9.67 \\
8.49 \\
8.48\end{array}$ & $\begin{array}{r}11.09 \\
9.52 \\
8.33 \\
8.32\end{array}$ & $\begin{array}{l}17.6 \pm 0.8^{e} \\
11.7 \pm 0.7^{e} \\
6.5 \pm 0.7^{f} \\
7.4 \pm 0.5^{e}\end{array}$ \\
\hline $\mathfrak{S}_{0}(1200 \mid \mathrm{A})_{\mathrm{AB}}$ & $\begin{array}{r}85.0 \\
300.0\end{array}$ & $\begin{array}{r}10.86 \\
6.49\end{array}$ & $\begin{array}{l}9.61 \\
5.71\end{array}$ & $\begin{array}{r}11.29 \\
7.65\end{array}$ & $\begin{array}{r}11.43 \\
7.63\end{array}$ & $\begin{array}{r}11.49 \\
7.77\end{array}$ & $\begin{array}{r}11.37 \\
7.63\end{array}$ & $\begin{array}{l}13.0 \pm 1.3^{g} \\
8.2 \pm 0.8^{g} \\
8.7 \pm 0.9^{h}\end{array}$ \\
\hline $\mathfrak{S}\left(\left.\begin{array}{c}0200 \\
2000\end{array}\right|_{\mathrm{A}} ^{\mathrm{A}}\right)_{\mathrm{AB}}$ & $\begin{array}{r}77.3 \\
293.0 \\
295.0\end{array}$ & $\begin{array}{l}0.110 \\
0.052 \\
0.052\end{array}$ & $\begin{array}{l}0.097 \\
0.041 \\
0.041\end{array}$ & $\begin{array}{l}0.134 \\
0.076 \\
0.076\end{array}$ & $\begin{array}{l}0.135 \\
0.077 \\
0.076\end{array}$ & $\begin{array}{l}0.137 \\
0.079 \\
0.079\end{array}$ & $\begin{array}{l}0.134 \\
0.076 \\
0.076\end{array}$ & $\begin{array}{l}0.14 \pm 0.01^{c} \\
0.080 \pm 0.008^{c} \\
0.077 \pm 0.01^{d}\end{array}$ \\
\hline $\mathfrak{S}\left(\left.\begin{array}{l}1000 \\
1200\end{array}\right|_{\mathrm{A}} ^{\mathrm{A}}\right)_{\mathrm{AB}}$ & 300.0 & -0.159 & -0.145 & -0.163 & -0.164 & -0.168 & -0.164 & $0.121 \pm 0.015^{h, i}$ \\
\hline $\mathfrak{S}\left(\left.\begin{array}{l}1010 \\
1200\end{array}\right|_{\mathrm{A}} ^{\mathrm{A}}\right)_{\mathrm{AB}}$ & $\begin{array}{r}85.0 \\
300.0\end{array}$ & $\begin{array}{l}-0.052 \\
-0.027\end{array}$ & $\begin{array}{l}-0.044 \\
-0.021\end{array}$ & $\begin{array}{l}-0.069 \\
-0.040\end{array}$ & $\begin{array}{l}-0.069 \\
-0.040\end{array}$ & $\begin{array}{l}-0.071 \\
-0.042\end{array}$ & $\begin{array}{l}-0.088 \\
-0.052\end{array}$ & $-0.04 \pm 0.004^{g}$ \\
\hline $\mathfrak{S}\left(\left.\begin{array}{l}1001 \\
1200\end{array}\right|_{\mathrm{A}} ^{\mathrm{A}}\right)_{\mathrm{AB}}$ & $\begin{array}{r}85.0 \\
300.0\end{array}$ & $\begin{array}{l}0.557 \\
0.316\end{array}$ & $\begin{array}{l}0.502 \\
0.265\end{array}$ & $\begin{array}{l}0.590 \\
0.416\end{array}$ & $\begin{array}{l}0.593 \\
0.416\end{array}$ & $\begin{array}{l}0.604 \\
0.428\end{array}$ & $\begin{array}{l}0.768 \\
0.536\end{array}$ & $\begin{array}{l}1.4 \pm 0.35^{g} \\
0.46 \pm 0.12^{j} \\
0.44 \pm 0.11 k\end{array}$ \\
\hline
\end{tabular}

${ }^{a}$ Ref. [87]

${ }^{b}$ Refs. [66], [71]

${ }^{c}$ Ref. [72]

${ }^{d}$ Ref. [75]

${ }^{e}$ Ref. [88]

${ }^{f}$ Ref. [89]

$g$ Ref. [73]; experimental uncertainty from Ref. [91]

${ }^{h}$ Ref. [74]; experimental uncertainty from Ref. [91]

${ }^{i}$ Only the absolute value has been reported for this effective cross section.

$j$ Refs. [73, 90]

${ }^{k}$ Refs. [73], [92] 
of disagreement between the calculated and measured values.

A different category of bulk properties that is frequently considered is provided by simple relaxation phenomena, such as vibrational, rotational, and nuclear spin magnetization relaxation, by the collisional broadening and shifting of infrared and Raman spectroscopic lines, and by the collision-broadening of the depolarized Rayleigh light scattering spectrum, on the one hand, and by the effects of external magnetic and electric fields on gas transport properties, on the other hand. Most of these bulk properties depend upon departures of the intermolecular interaction from isotropy. This is particularly true for rotational relaxation and nuclear spin magnetization relaxation, as well as for the effects of external magnetic fields on the transport properties of polyatomic gases. Vibrational relaxation, however, is governed primarily by the stretching dependence of the polyatomic species themselves, and necessitates a three-dimensional PES for its calculation. While the collision-broadened widths of infrared and Raman spectroscopic lines and of the depolarized Rayleigh light scattering spectrum depend mostly upon the anisotropy of the PES, the collisional shifting of the spectroscopic and Raman lines depends strongly upon both the monomer stretching dependence and the anisotropy of the PES.

The classic relaxation phenomenon is rotational relaxation, which is governed by the effective cross section $\mathfrak{S}(0001 \mid \mathrm{A})_{\mathrm{AB}}$. Until relatively recently, rotational relaxation data were only accessible experimentally through non-classical sound absorption measurements. With the exception of the hydrogen isotopes, their binary mixtures, and simple hydrides, such as $\mathrm{HF}, \mathrm{HCl}$, and $\mathrm{CH}_{4}$, these data are notoriously difficult to isolate from measurements of the sound absorption coefficient. Indeed, the only reliable rotational relaxation time (which is directly related to the rotational relaxation effective cross section) for $\mathrm{N}_{2}-\mathrm{He}$ mixtures 
is that determined by Holmes et al. [66] from their room temperature sound absorption measurements. More recently, rotational relaxation cross sections in the temperature range 10-20 K have also been investigated using free-jet expansions $[69,70,71]$. The earlier study by Aoiz et al. [69] reported a constant value of $15 \AA^{2}$ for $\mathfrak{S}(0001 \mid \mathrm{A})_{\mathrm{AB}}$ over the temperature range $10 \mathrm{~K} \leq T \leq 50 \mathrm{~K}$, while their later study [70] reported values of $\mathfrak{S}(0001 \mid \mathrm{A})_{\mathrm{AB}}$ ranging from $18 \AA^{2}$ at $10 \mathrm{~K}$ to $10.5 \AA^{2}$ at $50 \mathrm{~K}$. Maté et al. [71] gave values of $\mathfrak{S}(0001 \mid \mathrm{A})_{\mathrm{AB}}$ between 11.5 $\AA^{2}$ and $13.5 \AA^{2}$ over the temperature range $10 \mathrm{~K} \leq T \leq 20 \mathrm{~K}$. These values may be compared with the present CT values $33.1 \AA^{2}$ at $10 \mathrm{~K}$ and $20.2 \AA^{2}$ at $20 \mathrm{~K}$ obtained for the BCCD PES if we realize that at these very low temperatures CT values for $\mathfrak{S}(0001 \mid A)_{A B}$ may be overestimated by as much as $100 \%$ relative to values calculated quantum mechanically.

The relaxation phenomena most sensitive to anisotropies on the repulsive wall of the PES are the relaxation of nuclear spin magnetization, which is goverened by one or both of the effective cross sections $\mathfrak{S}^{\prime}(0100 \mid \mathrm{A})_{\mathrm{AB}}, \mathfrak{S}^{\prime}(0 \widehat{2} 00 \mid \mathrm{A})_{\mathrm{AB}}$ (depending, in the present case, upon which $\mathrm{N}_{2}$ isotopomer is present), and pressure-broadening of the depolarized Rayleigh light scattering spectrum, which is governed by the effective cross section $\mathfrak{S}_{\mathrm{DPR}} \equiv \mathfrak{S}(0 \widehat{2} 00 \mid \mathrm{A})_{\mathrm{AB}}$. For polyatomic-monatomic binary mixtures, $\mathfrak{S}^{\prime}(0 \widehat{2} 00 \mid \mathrm{A})_{\mathrm{AB}}$ and $\mathfrak{S}(0 \widehat{2} 00 \mid \mathrm{A})_{\mathrm{AB}}$ are identical, otherwise they differ by about $20 \%$. While it is unfortunate that no experimental data are available for the relaxation of nuclear spin magnetization in $\mathrm{N}_{2}-\mathrm{He}$ mixtures, data are available for the pressure-broadening of the depolarized Rayleigh light scattering spectrum $[89,88]$. We should take note, however, that values for $\mathfrak{S}(0 \widehat{2} 00 \mid \mathrm{A})_{\mathrm{AB}}$ are less reliable than nuclear magnetization relaxation cross section values, because it is more difficult to extract the DPR effective cross section unequivocally from the raw experimental data, especially for low temperatures. 
Because direct comparisons between experimental and calculated values of bulk relaxation phenomena are complicated by the lack of published experimental values (other than those to be found in figures embedded in the original publications), comparisons between theory and experiment are normally made between the calculated and experimentally-extracted values of the effective cross sections that determine the phenomena. Such a comparison can be found in Table 2, in which the relevant effective cross section values obtained using the present BCCD PES with $r$ fixed at $r_{0}$ are compared with the corresponding experimental values and with values obtained in Ref. [87] for the HT, MTE, and XC(fit) potential energy surfaces. For completeness, we have also included values for these relaxation effective cross sections obtained from the empirical ESMSV and M3SV potential energy surfaces.

Transport property magnetic field-effects, such as the viscomagnetic, thermomagnetic, and thermodiffusiomagnetic effects, involve both the effective cross sections determining the corresponding field-free (or ordinary) transport coefficient and additional effective cross sections related to the specific field-effect phenomenon. The viscomagnetic effect involves, in addition to the effective cross sections determining the ordinary shear viscosity, the relaxation effective cross section $\mathfrak{S}(0200 \mid \mathrm{A})_{\mathrm{AB}}$ and the production effective cross section $\mathfrak{S}\left(\left.{ }_{0200}^{2000}\right|_{\mathrm{A}} ^{\mathrm{A}}\right)_{\mathrm{AB}}$. Burgmans et al. [72] have obtained values for these effective cross sections from their viscomagnetic effect measurements. Similarly, the thermomagnetic effect involves the relaxation effective cross section $\mathfrak{S}_{0}(1200 \mid \mathrm{A})_{\mathrm{AB}}$ and the production effective cross sections $\mathfrak{S}\left(\left.{ }_{1200}^{1010}\right|_{\mathrm{A}} ^{\mathrm{A}}\right)_{\mathrm{AB}}$ and $\mathfrak{S}\left(\left.{ }_{1200}^{1001}\right|_{\mathrm{A}} ^{\mathrm{A}}\right)_{\mathrm{AB}}$ in addition to those effective cross sections determining the ordinary thermal conductivity. Because the thermodiffusiomagnetic effect is a cross-effect, it involves both the relaxation and production effective cross sections just listed for the thermomagnetic effect and an additional production effective cross section $\mathfrak{S}\left(\left.\begin{array}{l}1000 \\ 1200\end{array}\right|_{\mathrm{A}} ^{\mathrm{A}}\right)_{\mathrm{AB}}$ that is also 
associated with the diffusiomagnetic effect. Experimental values for most of these effective cross sections have been obtained by the Leiden group [73, 74, 92, 91].

Additional information on and expressions for the bulk transport properties and their magnetic field-effects can be found in Refs. [40] and [82].

\section{Summary and Conclusions.}

This paper reports on an improved three-dimensional version of the BCCD ab initio $\mathrm{N}_{2}-\mathrm{He}$ PES, obtained at the CCSD $(\mathrm{T})$ level of quantum supermolecule calculations and based upon 790 points, rather than upon 370 points as in the earlier version reported in Ref. [12]. The present version of the BCCD PES has been fitted, as previously [12], to a three-dimensional functional representation via the reproducing-kernel Hilbert-space method [14]. In particular, the dependence on the $\mathrm{N}-\mathrm{N}$ separation $r$ has been better delineated by including four $r$-values, in addition to the equilibrium separation $r_{0}=2.0743 a_{0}$ for the $\mathrm{N}_{2}$ ground $(v=0)$ vibrational state, in a restricted interval in the neighborhood of $r_{0}$. Also, a more accurate representation of the short- and long-range components of the PES has been obtained by extending the range of (interspecies distance) $R$-values inward from $3 a_{0}$ to $2 a_{0}$ and outward from $12 a_{0}$ to $20 a_{0}$. The number of Jacobi angles $\theta$ was unchanged, as the angular dependence of the PES is not particularly strong. Figures 1 and 2 illustrate these improvements to the BCCD PES.

The ability of the present version of the $\mathrm{N}_{2}-\mathrm{He}$ BCCD PES to deal with three types of phenomena has been tested against experiment, with results obtained using the previous version [12] of this PES where relevant, and with results obtained using several other recent 
$\mathrm{N}_{2}-\mathrm{He}$ potential energy surfaces. The phenomena that have been considered here are: molecular beam scattering, both state-to-state and total differential scattering, and total integral scattering; collisional quenching of the first excited vibrational level $(v=1)$ of ${ }^{14} \mathrm{~N}_{2}$ by both ${ }^{3} \mathrm{He}$ and ${ }^{4} \mathrm{He}$; traditional bulk properties, including the interaction second virial coefficient and binary mixture transport properties (diffusion, shear viscosity, thermal conductivity). The potential energy surfaces considered here are those identified by the acronyms ESMSV [1], M3SV [2], both of which are fully empirical potential energy surfaces determined from fits to experimental data, XC(fit) [24], a semi-empirical PES determined from a combination of $a b$ initio and experimental data, and HT [6, 8], MTE [7], both of which are, like the previous and present versions of the BCCD PES, fully ab initio surfaces. In addition, we have included occasional comparisons with results obtained using the overly anisotropic semi-empirical HFD1 PES of Fuchs et al. [37], to help elucidate the role of PES anisotropy.

With the exception of the BCCD PES and the extended HT PES of Reid et al. [8], all potential energy surfaces considered here are two-dimensional, depending only upon the Jacobi coordinates $R$ and $\theta$ for fixed $\mathrm{N}-\mathrm{N}$ separation $r \equiv r_{0}=2.0743 a_{0}$. For this reason, calculations of all molecular beam scattering cross sections and bulk properties other than the vibrational quenching cross section using the present BCCD PES have been carried out with $r$ fixed at $r_{0}$.

Comparison of the predictions of the present BCCD PES with both the experimental state-to-state and total differential (scattering) cross sections (DCS) and the predictions of other potential energy surfaces have been presented in Figs. 3 and 4. Because the total DCS and ICS are largely determined by the isotropic component of a PES, they do not provide a particularly good means of differentiating amongst the various potential energy surfaces 
considered here, as the main differences are in the anisotropies, especially on the repulsive wall. This can be seen clearly from Fig. 3, in which the calculated values obtained using the various potential energy surfaces considered here are rather difficult to disentangle. The state-to-state differential cross sections show greater differentiation amongst these potential energy surfaces due to their strong dependence on the anisotropic components of the PES. As can be seen from Fig. 4, the differences are particularly marked in the cases of the 0 $\rightarrow 2$ (similarly, the $1 \rightarrow 3$ ) rotationally inelastic differential cross section at large centerof-mass scattering angles $\vartheta$ : the $\vartheta$ dependence associated with the empirical ESMSV PES and M3SV PES (nearly indistinguishable) differs markedly from that associated with the semi-empirical XC(fit) PES and the present BCCD PES (which show rather similar, though still distinguishable, behavior). We note that the $\vartheta$ dependence of the $0 \rightarrow 2$ DCS associated with each of the HT and MTE potential energy surfaces is very similar to that shown for the BCCD PES.

Because the behavior of the state-to-state differential cross section calculations for large values of the scattering angle raised a question about the nature of the differences between the empirically determined ESMSV [1] and M3SV [2] potential energy surfaces, on the one hand, and the semi-empirical $[24,37]$, and the present BCCD and other $[6,7,8]$ ab initio potential energy surfaces, on the other hand, Nearside-Farside (NF) analyses were carried out in order to compare the potential energy surfaces of Refs. [1, 2, 6, 7, 24], and [37]. NF calculations provide the only tool that allows a correlation to be established between variations in the structures of state-to-state differential cross sections and differences in competing potential energy surfaces. The results of these analyses are shown in Figs. 6-9. Calculations of the $0 \rightarrow 2,1 \rightarrow 3$, and $0 \rightarrow 4$ state-to-state DCS 
were carried out for total energies $220 \mathrm{~cm}^{-1}$ and $568 \mathrm{~cm}^{-1}$, corresponding approximately to the experimental collision energies of Refs. [19] and [1], respectively. Because scattering at these total energies primarily probes the repulsive wall of a PES, calculations were also carried out for total energies $13 \mathrm{~cm}^{-1}$ and $21 \mathrm{~cm}^{-1}$ in order to probe the well region of the PES. No information additional to that obtained from NF analyses of the $0 \rightarrow 2$ DCS could be extracted from the NF analyses of the $1 \rightarrow 3$ DCS.

For total energies $220 \mathrm{~cm}^{-1}$ and $568 \mathrm{~cm}^{-1}$, the final value of the $0 \rightarrow 2$ DCS is determined largely by the nearside component for scattering angles beyond $60^{\circ}$, and shows significant $\mathrm{NF}$ interference oscillations for scattering angles between $0^{\circ}$ and $60^{\circ}$ (corresponding to the experimental measurements). The MTE PES and the present BCCD PES behave rather similarly at both total energies (as does the HT PES, not shown in the figures). Both the HFD1 PES and the empirical M3SV and ESMSV potential energy surfaces exhibit centerof-mass scattering angle dependences that differ from that shown by the $a b$ initio potential energy surfaces, although the HFD1 PES does show qualitatively, but not quantitatively, similar behavior to them at both total energies. For total energy $220 \mathrm{~cm}^{-1}$ the $0 \rightarrow 2$ DCS calculated using the M3SV (equivalently, the ESMSV) PES behaves similarly as a function of $\vartheta$ to the $0 \rightarrow 2$ DCS obtained using the other potential energy surfaces for angles smaller than $60^{\circ}$, but it does not fall off as rapidly at large angles, with the consequence that it is considerably larger for $\vartheta$ values in excess of $120^{\circ}$. A similar behavior is not observed for total energy $568 \mathrm{~cm}^{-1}$. This suggests that the nature of the anisotropy on the low repulsive wall of the M3SV (ESMSV) PES differs significantly from that of the three ab initio potential energy surfaces. As can be seen from Fig. 9, a proper experimental test of the long-range parts of these potential energy surfaces will require either scattering measurements at collision 
energies of the order of $5-10 \mathrm{~cm}^{-1}$, where distinctly different oscillatory structures can be found for the various potential energy surfaces examined here, or the determination of the spectrum of the $\mathrm{He}-\mathrm{N}_{2}$ van der Waals dimer.

The present BCCD PES does not give as good agreement with the temperature dependence of the $\mathrm{N}_{2}-\mathrm{He}$ interaction second virial coefficient as the XC(fit) PES (which employed some of the most accurate virial data [47] in its determination). As can be seen from Table I, however, it does give a level of agreement with these data that is comparable to that obtained for the HT PES, and is only slighyly poorer than that obtained for the MTE PES.

Calculation of the energy dependence of the vibrational relaxation cross sections and of the vibrational quenching rate for the $v=1$ state of $\mathrm{N}_{2}$ requires a stretching-dependent PES. Both the previous [12] and the present versions of the BCCD PES are fully threedimensional, as is the Reid et al. [8] extension of the original HT PES of Hu and Thakkar [6]. Reid et al. [8] obtained good agreement between their experimental vibrational quenching rates and values calculated from their extension of the HT PES to include the dependence of the $\mathrm{N}_{2}-\mathrm{He}$ interaction energy on the $\mathrm{N}-\mathrm{N}$ (intramolecular) separation. Values for the vibrational quenching cross section of $\mathrm{N}_{2}(v=1, j=0)$ by collisions with ${ }^{3} \mathrm{He}$ obtained from the previous version [12] of the BCCD PES agreed with those obtained from the full HT PES for total energies of $200 \mathrm{~cm}^{-1}$ and above, but for lower total energies differed by as much as two orders of magnitude. This difference in quenching cross section values was one of the major motivations behind the present extended and improved BCCD PES. As shown in Figs. 11 and 12 , there is now considerably better agreement between the two sets of calculations and very good agreement between the present calculations and the experimental vibrational quenching rates of Ref. [8], especially for the ${ }^{3} \mathrm{He}^{-}{ }^{14} \mathrm{~N}_{2}$ case. 
Because there was a significantly larger difference between the ${ }^{4} \mathrm{He}^{-14} \mathrm{~N}_{2}$ calculations of Ref. [8], which are based upon coupled-states (CS) calculations, and the present results, which are full quantum close-coupled (CC) calculations, a CC calculation of the ${ }^{4} \mathrm{He}^{-}$ ${ }^{14} \mathrm{~N}_{2}(v=1)$ quenching cross section was performed using the full HT PES. As can be seen from Fig. 11, the CC calculations using the full HT PES are in much better agreement with the present calculations using the BCCD PES than are the CS calculations reported in Ref. [8]. This indicates that great care must be taken when employing CS approximation calculations at such low total energies.

It remains possible that the agreement between the $\mathrm{CC}$ results obtained with the present full BCCD and HT potential energy surfaces may be fortuitous, however, as the full HT surface [8] does not include ab initio points in the repulsive region of the PES for values of $R$ less than 4 bohr.

Classical trajectory calculations using the BCCD PES for $r$ fixed at $r_{0}$ have been carried out to give a set of 43 effective cross sections for temperatures ranging from $50 \mathrm{~K}$ up to $1100 \mathrm{~K}$. These effective cross sections have been employed to calculate the mixture diffusion, shear viscosity, and thermal conductivity coefficients for comparison with a total of 144 experimental values (60 diffusion, 81 shear viscosity, 3 thermal conductivity). The level of agreement between theory and experiment for these transport properties has been summarized in Table I in terms of dimensionless root mean square deviation (DRMSD) values. It should be noted that, even though the DRMSD values for all potential energy surfaces under consideration here exceed 1 , the reason is that the uncertainties for much of the transport coefficient data, typically stated to be less than $\pm 1 \%$, and in many cases, smaller than $\pm 0.5 \%$, may be underestimated. 
Bulk relaxation phenomena are typically much more difficult to determine with great accuracy, and because the primary effective cross sections, especially production effective cross sections, must often be teased from the measurements, the experimental uncertainties for some of them can be as high as $\pm 20 \%$ [91]. The calculated and experimentally-determined values of these effective cross sections have been compared in Table II. We have also included effective cross section values reported for the $\mathrm{XC}(\mathrm{fit}), \mathrm{HT}$, and $\mathrm{MTE}$ potential energy surfaces in Ref. [87] for comparison. DMSRD values $1.75,1.76$, and 1.79 , respectively, for the XC(fit), MTE, and HT potential energy surfaces have been reported in Ref. [87] for the set of effective cross section values appearing in Table II, excluding the value for $\mathfrak{S}(0 \widehat{2} 00 \mid \mathrm{A})_{\mathrm{AB}}$ for temperature $120 \mathrm{~K}$; the corresponding DRMSC value for the BCCD PES is 1.90. This does not represent a significant difference from the other high-level potential energy surfaces.

The present BCCD $\mathrm{N}_{2}-\mathrm{He}$ PES predicts bulk phase virial and transport coefficients over the temperature range $77.3 \mathrm{~K}$ to $750 \mathrm{~K}$ with an accuracy that is, on the one hand, only slightly poorer than that obtained using the $\mathrm{XC}$ (fit) [24] and $\mathrm{MTE}$ [7] potential energy surfaces, and is, on the other hand, significantly better than that achievable with the HT PES $[6,8]$. Moreover, the three-dimensional version of the present BCCD PES has the most accurate representation of the stretching dependence available; it predicts the vibrational relaxation quenching rate for ${ }^{14} \mathrm{~N}_{2}(v=1)$ colliding with ${ }^{3} \mathrm{He}$ and ${ }^{4} \mathrm{He}$ at least as well as the full (i.e., stretching dependent) HT PES [8]. At the same time, it gives only marginally less accurate descriptions of other, non-stretching-dependent, bulk relaxation phenomena.

\section{Acknowledgments.}

The quantum close--coupled scattering calculations carried out by JSFS have been made 
possible by the facilities of the Shared Hierarchical Academic Research Computing (SHARCNET, www.sharcnet.ca) and by access to FLEXOR, a high-performance computing facility purchased through funding provided by the Canada Foundation for Innovation (CFI). Calculations performed by TS have been performed using the computing resources available at IDRIS in Paris as well as in pole M3PEC Université Bordeaux 1. This work has also been supported in part by an NSERC of Canada Discovery Grant to FRWM. The authors are grateful to Professor Alan Dickinson (Newcastle, UK) for a number of insightful comments on our original manuscript. 


\section{References}

[1] L. Beneventi, P. Casavechia, and G. G. Volpi, J. Chem. Phys. 86, 7011 (1986).

[2] F. A. Gianturco, M. Venanzi, R. Candori, F. Pirani, F. Vecchiocattivi, A. S. Dickinson, and M. S. Lee, Chem. Phys. 109, 417 (1986); Erratum, ibid 113, 166 (1987).

[3] K. T. Tang, and J. P. Toennies, J. Chem. Phys. 80, 3726 (1984).

[4] M. S. Bowers, K. T. Tang, and J. P. Toennies, J. Chem. Phys. 88, 5465 (1988).

[5] L. Beneventi, P. Casavecchia, G. G. Volpi, C. C. Wong, F. R. W. McCourt, G. C. Corey, and D. Lemoine, J. Chem. Phys. 95, 5827 (1991).

[6] C.-H. Hu and A. J. Thakkar, J. Chem. Phys. 104, 2541 (1996).

[7] K. Patel, P. R. Butler, A. M. Ellis, and M. D. Wheeler, J. Chem. Phys. 119, 909 (2003).

[8] J. P. Reid, A. J. Thakkar, P. W. Barnes, E. F. Archibong, H. M. Quiney, and C. J. S. M. Simpson, J. Chem. Phys. 107, 2329 (1997).

[9] M. M. Maricq, E. A. Gregory, C. T. Wickham-Jones, D. J. Cartwright, and C. J. S. M. Simpson, Chem. Phys. 75, 347 (1983).

[10] J. M. Hutson and S. Green, MOLSCAT Parallel Computer Code developed from Version 12 (1993) of the serial code by I. J. Bush, distributed by Collaborative Computational Project No. 6 of the Engineering and Physical Sciences Research Council $(\mathrm{UK})$. 
[11] N. Balakrishnan and A. Dalgarno, J. Phys. Chem. A 105, 2348 (2001).

[12] T. Stoecklin, A. Voronin, and J. C. Rayez, Phys. Rev. 66, 042703 (2002).

[13] N. C. Handy, J. A. Pople, M. Head-Gordon, K. Raghavacheri, and G. W. Trucks, Chem. Phys. Lett. 164, 185 (1989).

[14] T. S. Ho and H. Rabitz, J. Chem. Phys. 104, 2584 (1996).

[15] The Newmat code, developed and maintained by T. Stoecklin, solves the closecoupling equations for inelastic collisions in the space-fixed frame.

[16] E. B. Steckel, R. B. Walker, and J. C. Light, J. Chem. Phys. 69, 3518 (1978); R. W. Anderson, J. Chem. Phys. 77, 4431 (1982).

[17] G. McBane, PMP MOLSCAT, a parallel version of MOLSCAT version 14 available at http://faculty.gvsu.edu/mcbaneg/pmpmolscat, Grand Valley State University (2005).

[18] S. Green and J. M. Hutson, DCS computer code, version 2.0 (1996), distributed by Collaborative Computational Project No. 6 of the Engineering and Physical Sciences Research Council (UK).

[19] M. Faubel, K. H. Kohl, J. P. Toennies, K. T. Tang, and Y. Y. Yung, J. Chem. Soc. Faraday Disc. 73, 205 (1982).

[20] S. Schlemmer, Hochaufgelöste Molekularstrahluntersuchungen der inelastischen und reaktiven Streuung kleiner Moleküle, Ph.D. thesis, University of Göttingen, 1991. 
[21] M. Faubel, in Status and Future Developments in the Study of Transport Properties, NATO ASI Series C, 361, 73 (1992), eds. W. A. Wakeham, A. S. Dickinson, F. R. W. McCourt, and V. Vesovic (Kluwer, Dordrecht).

[22] H. P. Butz, R. Feltgen, H. Pauly, H. Vehmeyer, and R. M. Yealland, Z . Phys. 247, $60(1971)$

[23] R. Candori, F. Pirani, F. Vecchiocattivi, F. A. Gianturco, U. T. Lamanna, and G. Petrella, Chem. Phys. 92, 345 (1985); Erratum, ibid 97, 464 (1985).

[24] A. K. Dham, F. R. W. McCourt, and A. S. Dickinson, J. Chem. Phys., 127054302 (2007).

[25] The Nearside-Farside method has been implemented in the Newmat code of Ref. [15] following recommendations of McCabe et al. in Ref. [31].

[26] P. McCabe and J. N. L. Connor, J. Chem. Phys. 104, 2297 (1995).

[27] J. Bendtsen, J. Raman Spectrosc. 2, 133 (1974).

[28] F. A. Gianturco and A. Palma, J. Phys. B 18, L519 (1985).

[29] A. S. Dickinson, Comp. Phys. Commun. 17, 51 (1979).

[30] D. J. Kouri, in Atom-Molecule Collision Theory: A Guide for the Experimentalist, R. B. Bernstein, ed. Plenum Press, New York, 1979, p. 31.

[31] P. McCabe, J. N. L. Connor, and D. Sokolovski, J. Chem. Phys. 1085695 (1998).

[32] P. McCabe, J. N. L. Connor, and D. Sokolovski, J. Chem. Phys. 1145194 (2000). 
[33] T. W. J. Whiteley, C. Noli, and J. N. L. Connor, J. Phys. Chem. 1052792 (2001).

[34] R. C. Fuller, Phys. Rev. C 12, 1561 (1975).

[35] P. J. Hatchell, Phys. Rev. C 40, 27 (1989).

[36] G. Guillon and T. Stoecklin, Eur. J. Phys. D 39, 359 (2006).

[37] R. R. Fuchs, F. R. W. McCourt, A. J. Thakkar, and F. Grein, J. Phys. Chem. 88, 2036 (1984).

[38] H. E. Cox, F. W. Crawford, E. B. Smith, and A. R. Tindell, Mol. Phys. 40, 705 (1980).

[39] G. C. Maitland, V. Vesovic, and W. A. Wakeham, Mol. Phys. 54, 287 (1985).

[40] F. R. W. McCourt, J. J. M. Beenakker, W. E. Köhler, and I. Kuščer, Nonequilibrium Phenomena in Polyatomic Gases. I. Dilute Gases (Oxford University Press, Oxford, $1990)$

[41] F. R. W. McCourt, J. J. M. Beenakker, W. E. Köhler, and I. Kuščer, Nonequilibrium Phenomena in Polyatomic Gases. II. Cross Sections, Scattering and Rarefied Gases (Oxford University Press, Oxford, 1992).

[42] C. M. Knobler, J. J. M. Beenakker, and H. F. P. Knaap, Physica 25, 909 (1959).

[43] R. J. Witansky, and J. G. Miller, J. Amer. Chem. Soc. 85, 282 (1963).

[44] F. B. Canfield, T. W. Leland, Jr., and A. Kobayashi, Adv. Cryogen. Eng. 8, 146 $(1963)$.

[45] P. S. Ku, and B. F. Dodge, J. Chem. Eng. Data 12, 158 (1967). 
[46] K. R. Hall, and F. B. Canfield, Physica 47, 219 (1969).

[47] J. Brewer, and G. W. Vaughn, J. Chem. Phys. 50, 2960 (1969).

[48] T. N. Bell, and P. J. Dunlop, Chem. Phys. Lett. 84, 99 (1981).

[49] B. Schramm, and A. Büchner, Chem. Phys. Lett. 98, 118 (1983).

[50] R. E. Walker, and A. A. Westenberg, J. Chem. Phys. 29, 1139, 1147 (1958)

[51] S. L. Seager, L. R. Geertson, and J. C. Giddings, J. Chem. Eng. Data 8, 168 (1963).

[52] B. A. Ivakin, and P. E. Suetin, Sov. Phys. - Tech. Phys. 9, 866 (1964).

[53] S. P. Wasik, and K. E. McCulloh, J. Res. Nat. Bur. Stand. 73A, 207 (1969).

[54] C. S. Ellis and J. N. Holsen, Indust. \& Eng. Chem. Fundamentals 8, 787 (1969).

[55] R. D. Trengove, and P. J. Dunlop, Physica A 115, 339 (1982).

[56] R. D. Trengove, K. R. Harris, H. L. Robjohn, and P. J. Dunlop, Physica A 131, 506 (1985); erratum, Physica A 144, 254 (1987); note that the coefficients $a_{2}$ as given in Table III of the erratum are a factor 10 too small (private communication from P. J. Dunlop to A. S. Dickinson, 1990).

[57] A. S. M. Wahby, and J. Los, Physica C 145, 69 (1987).

[58] J. Kestin, Y. Kobayashi, and R. T. Wood, Physica 32, 1065 (1966) .

[59] J. T. F. Kao and R. Kobayashi, J. Chem. Phys. 47, 2836 (1967).

[60] J. Kestin, S. T. Ro, and W. A. Wakeham, J. Chem. Phys. 56, 4036 (1972). 
[61] R. D. Fleeter, J. Kestin, R. Paul, and W. A. Wakeham, Physica A 108, 371 (1981).

[62] H. van Ee, Ph.D. thesis, University of Leiden, 1966.

[63] W. A. Oost, J. Los, H. Cauwenbergh, and W. Van Dael, Physica 62, 409 (1972).

[64] J. M. Symons, M. L. Martin, and P. J. Dunlop, J. Chem. Soc. Faraday Trans. I 75, $621(1979)$

[65] W. L. Taylor, J. Chem. Phys. 105, 8333 (1996).

[66] R. Holmes, G. R. Jones, N. Pusat, and W. Tempest, Trans. Faraday Soc. 58, 2342 $(1962)$

[67] A. E. Belikov, and R. G. Sharafutdinov, Chem. Phys. Lett. 241, 209 (1995)

[68] A. E. Belikov, R. G. Sharafutdinov, and A. V. Storozhev, Chem. Phys. 213, 319 (1996).

[69] F. J. Aoiz, L. Bañares, V. J. Herrero, B. Martínez-Haya, M. Menéndez, P. Quintana, L. Ramonat, I. Tanarro, and E. Verdasco, Vacuum 64, 417 (2002).

[70] F. J. Aoiz, L. Bañares, V. J. Herrero, B. Martínez-Haya, M. Menéndez, P. Quintana, L. Ramonat, I. Tanarro, and E. Verdasco, Chem. Phys. Lett. 367, 500 (2003).

[71] B. Maté, F. Thibault, A. Ramos, G. Tejeda, J. M. Fernández, and S. Montero, J. Chem. Phys. 118, 4477 (2003).

[72] A. L. J. Burgmans, P. G. van Ditzhuyzen, and H. F. P. Knaap, Z. Naturforsch. a 28, 849 (1973). 
[73] J. P. J. Heemskerk, G. F. Bulsing, and H. F. P. Knaap, Physica 71, 515 (1974).

[74] G. W. 't Hooft, E. Mazur, J. M. Bienfait, L. J. F. Hermans, H. F. P. Knaap, and J. J. M. Beenakker, Physica A 98, 41 (1979).

[75] J. N. Breunese, F. W. Gödecke, L. J. F. Hermans, and J. J. M. Beenakker, Physica A 126, $82(1984)$.

[76] R. T Pack, J. Chem. Phys. 78, 7217 (1983).

[77] T. Colbert and W. H. Miller, J. Chem. Phys. 96, 1982 (1992).

[78] W. E. Köhler and J. Schaefer, Physica A 120, 185 (1983).

[79] J. Schaefer and W. E. Köhler, Physica A 129, 469 (1985).

[80] F. R. W. McCourt, D. Weir, M. Thachuk, and G. B. Clark, Mol. Phys. 103, 45 (2005).

[81] J. M. Hutson and F. R. W. McCourt, J. Chem. Phys. 80, 1135 (1984).

[82] F. R. W. McCourt, Chapter 28, 712-751, Handbook of Molecular Physics and Quantum Chemistry. Ed. S. Wilson, Vol. 3: Molecules in the Physico-chemical Environment: Spectroscopy, Dynamics and Bulk Properties (John Wiley, New York, 2003).

[83] A. S. Dickinson and M. S. Lee, J. Phys. B 18, 4177 (1985).

[84] A. S. Dickinson and M. S. Lee, J. Phys. B 19, 3091 (1986).

[85] B. Schramm, Faraday Disc. Chem. Soc. 73, 283 (1982). 
[86] See EPAPS Document No. E-JCPSA6-127-027727 for electronic files that contain tables of effective cross sections for the BCCD PES and all others discussed in this paper. This document may be retrieved via the EPAPS homepage (http://www.aip.org/pubservs/epaps.html) or from ftp.aip.org in the directory /epaps/. See the EPAPS homepage for additional information.

[87] J. Sanchez-Fortún Stoker, A. K. Dham, F. R. W. McCourt, and A. S. Dickinson, J. Chem. Phys. (in preparation).

[88] R. J. van den Oord, W. Mischke, and J. J. M. Beenakker, Physica A 139 (1986).

[89] R. A. J. Keijser, K. D. van den Hout, and H. F. P. Knaap, Physica 76, 577 (1974).

[90] J. P. J. Heemskerk, F. G. van Kuik, H. F. P. Knaap, and J. J. M. Beenakker, Physica $71,484(1974)$

[91] H. van Houten, L. J. F. Hermans, and J. J. M. Beenakker, Physica A 131, 64 (1985)

[92] B. J. Thijsse, W. A. P. Denissen, L. J. F. Hermans, H. F. P. Knaap, and J. J. M. Beenakker, Physica A 97, 467 (1979). 


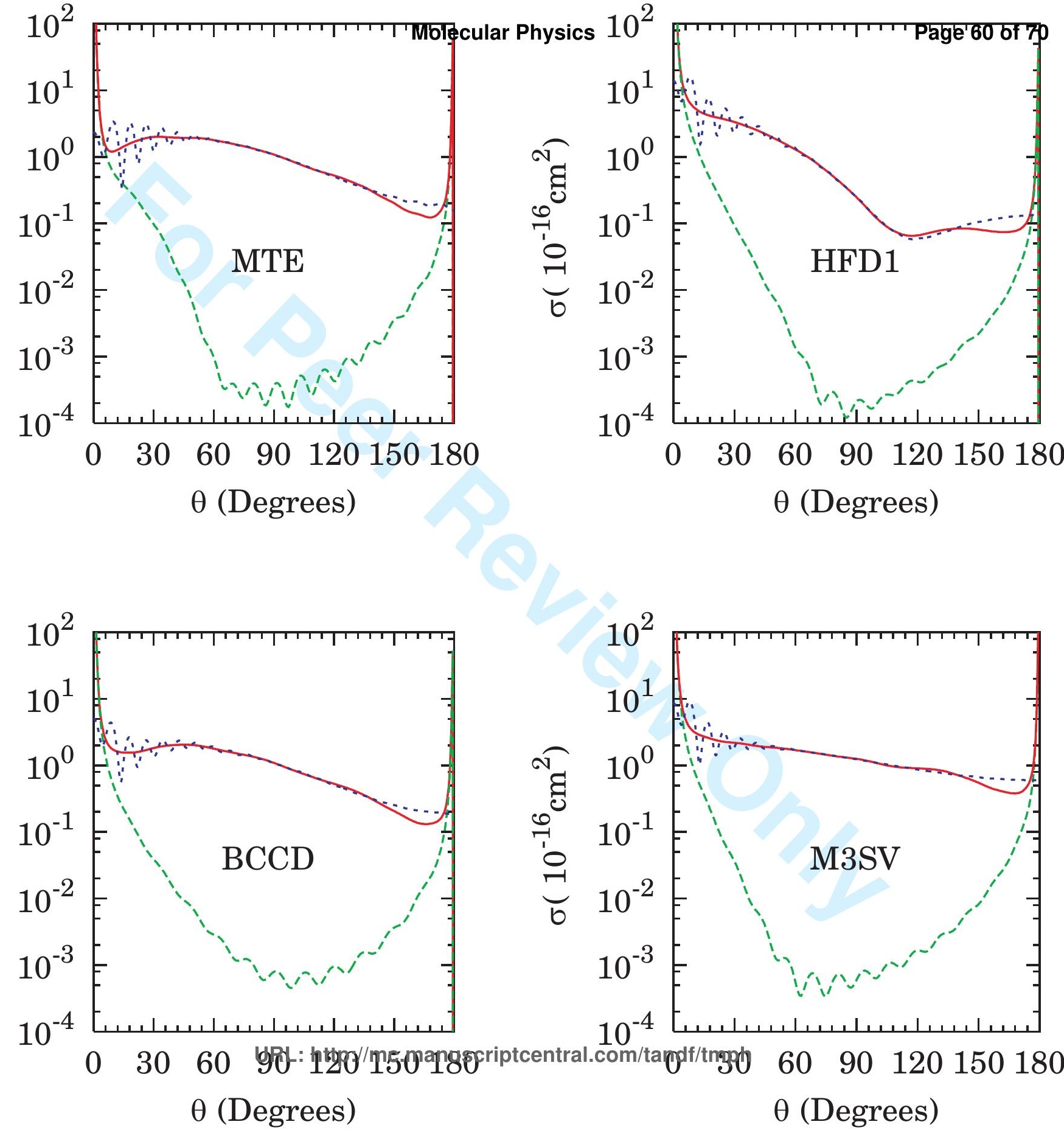




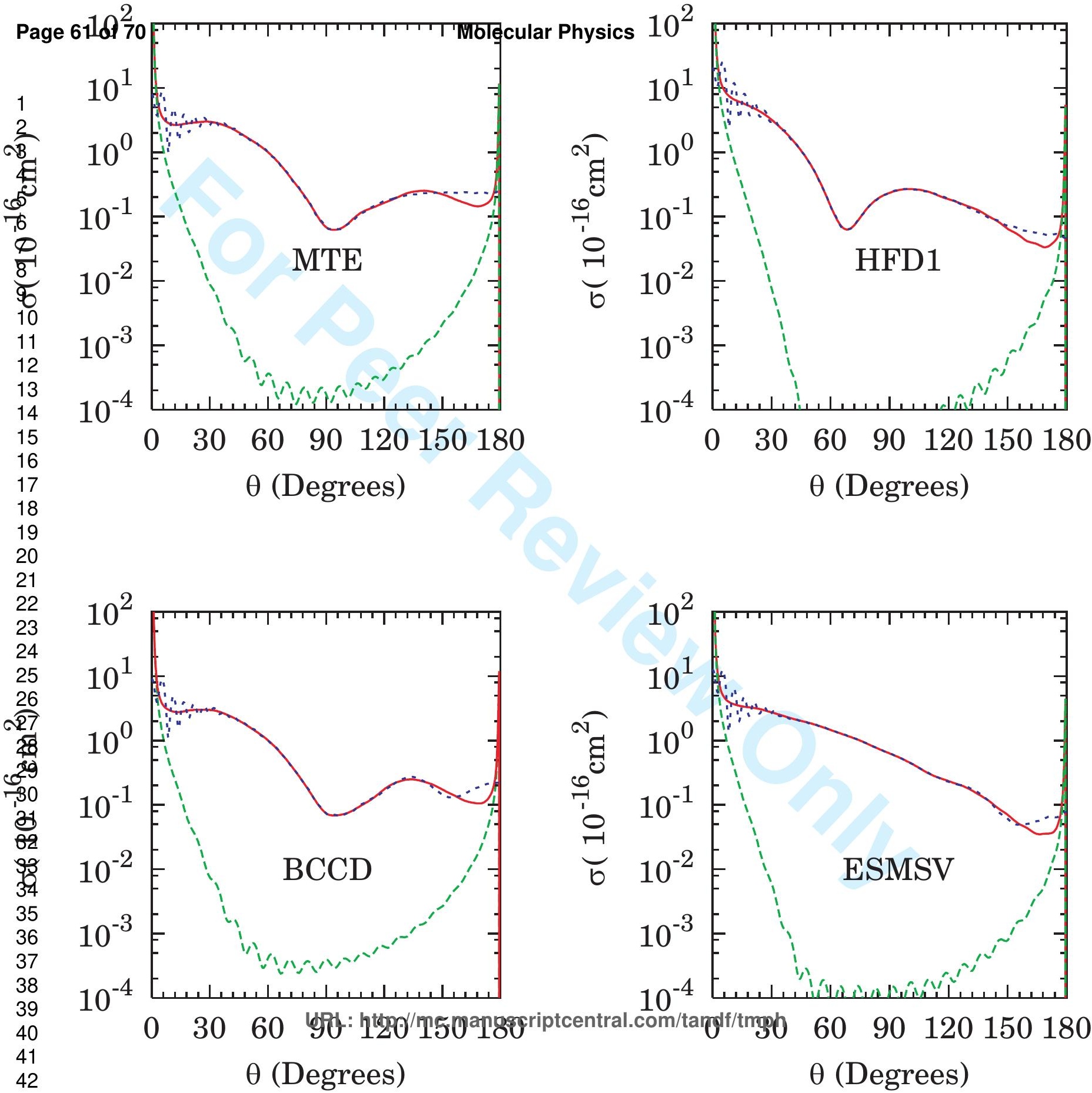




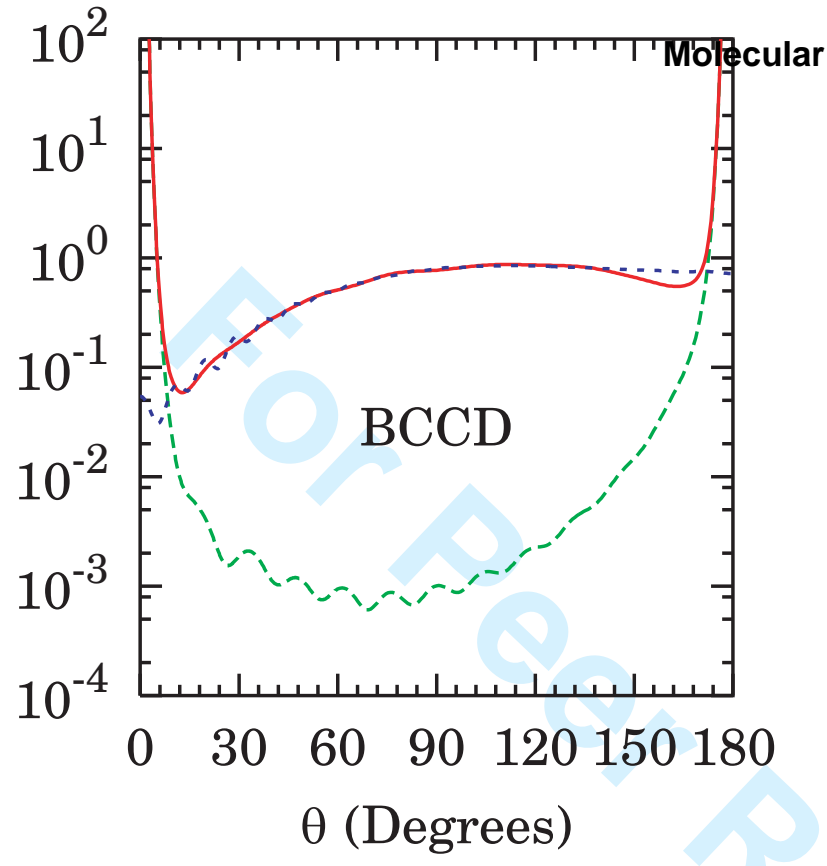

Physics $10^{2}$

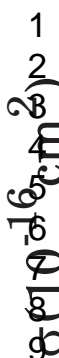
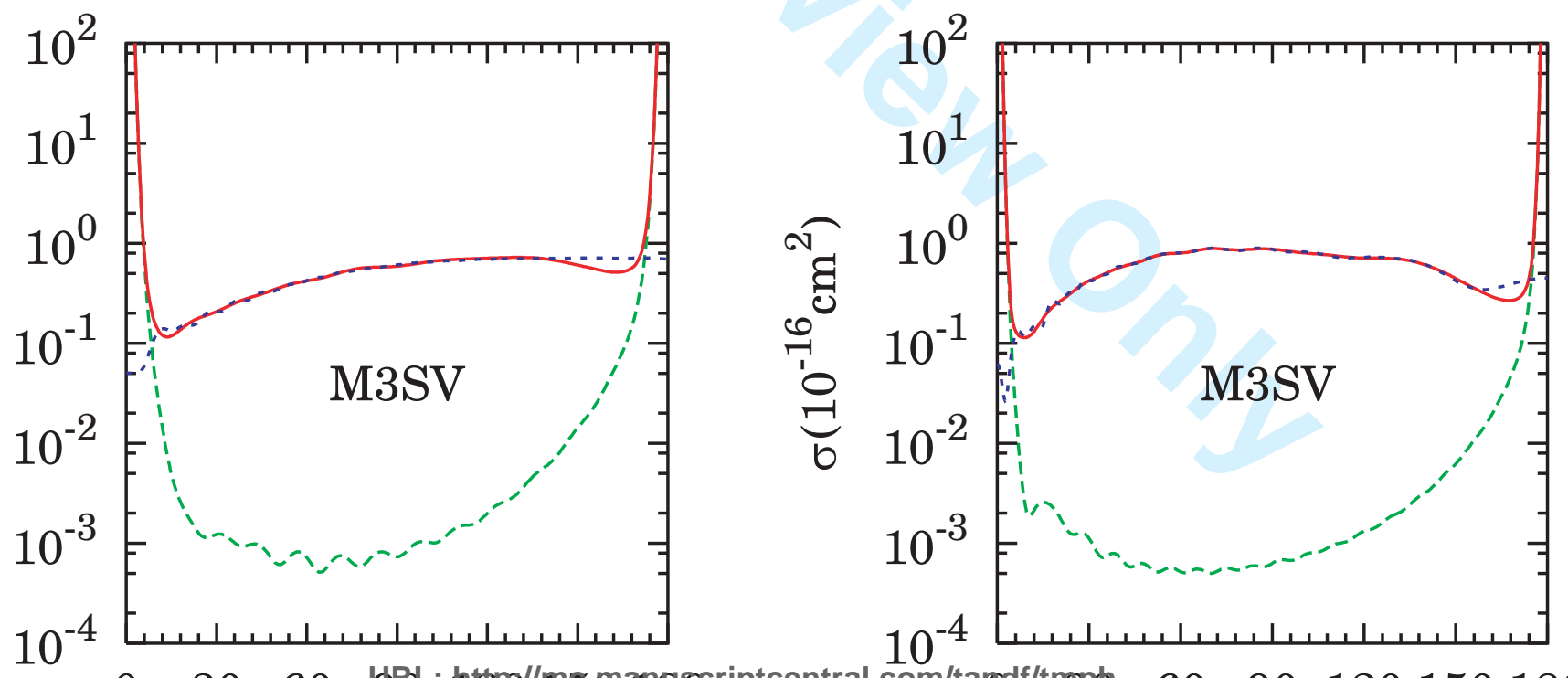

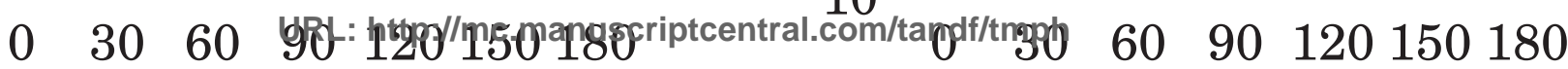




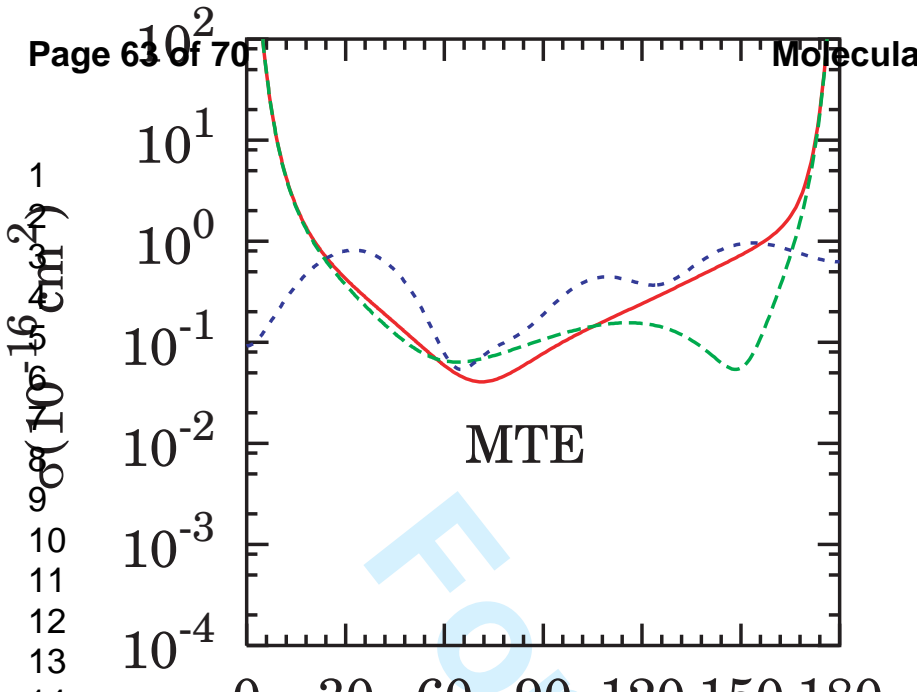




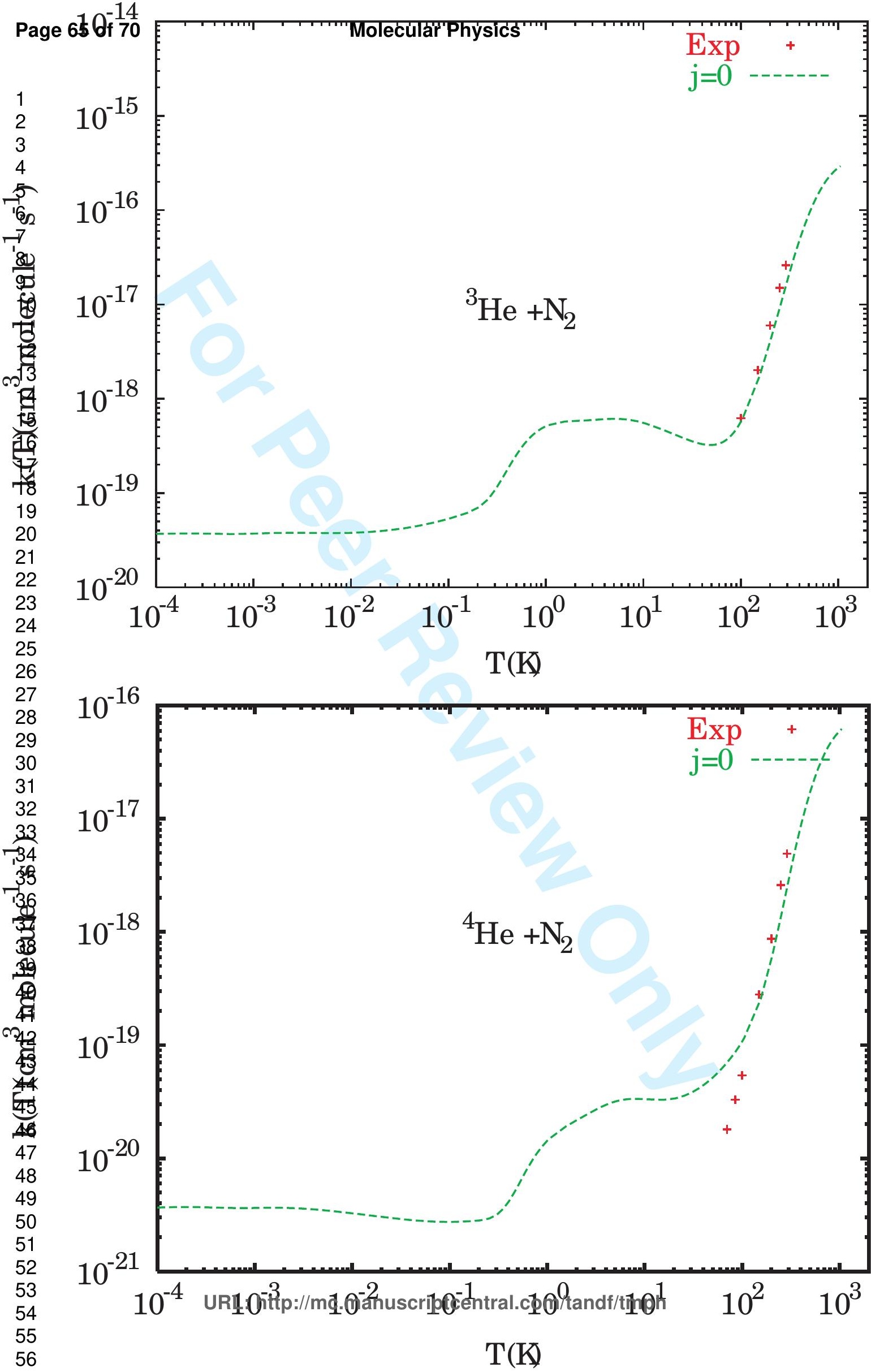




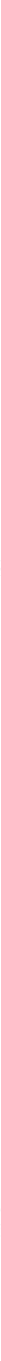




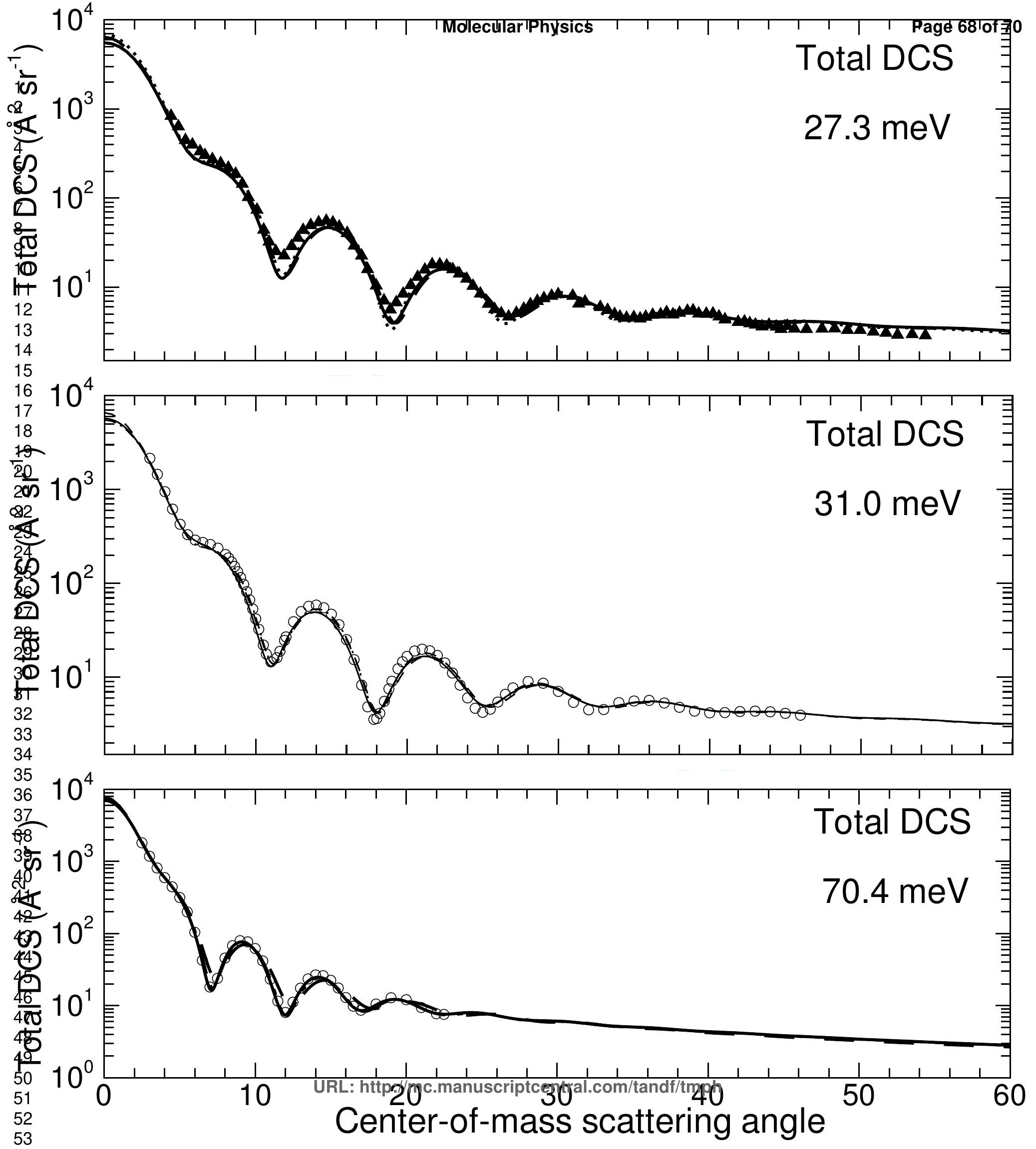




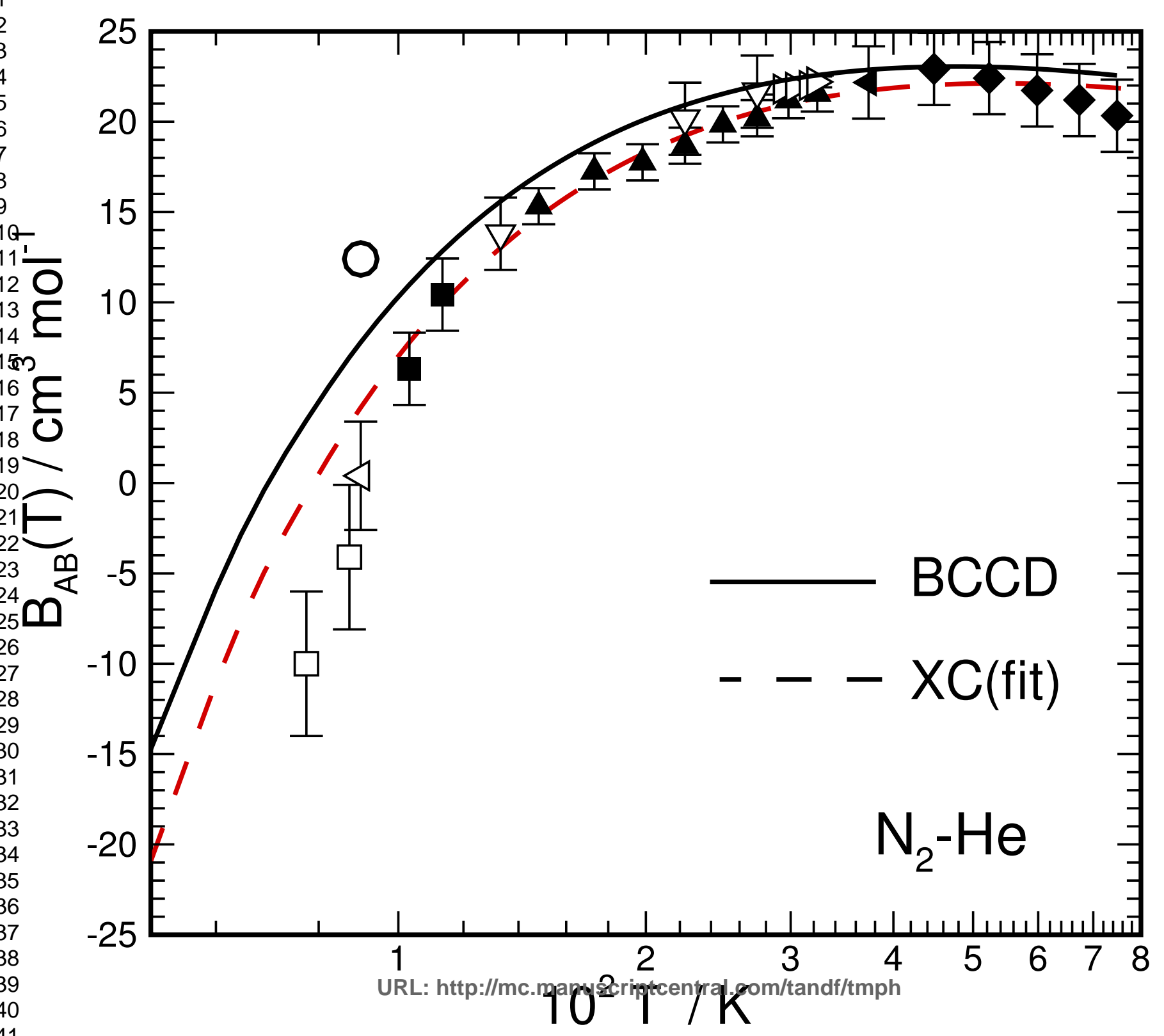

